\title{
Redetermination of the strong-interaction width in pionic hydrogen
}

\author{
A. Hirtl ${ }^{1,11}$, D. F. Anagnostopoulos ${ }^{2}$, D. S. Covita ${ }^{3,4,12}$, H. Fuhrmann ${ }^{1}$, H. Gorke ${ }^{5}$, D. Gotta ${ }^{6, a}$, A. Gruber ${ }^{1,13}$, \\ M. Hennebach ${ }^{6,14}$, P. Indelicato ${ }^{7}$, Th. S. Jensen ${ }^{3,8,15}$, E.-O. Le Bigot ${ }^{7}$, Y.-W. Liu ${ }^{3,16}$, V. E. Markushin ${ }^{3, b}$, J. Marton ${ }^{1}$, \\ M. Nekipelov ${ }^{6,17}$, J. M. F. dos $\operatorname{Santos}^{4}$, L. M. Simons ${ }^{3}$, Th. Strauch ${ }^{6}$, M. Trassinelli ${ }^{9}$, J. F. C. A. Veloso ${ }^{10}$, \\ J. Zmeskal ${ }^{1}$ \\ ${ }^{1}$ Stefan Meyer Institute for Subatomic Physics, Austrian Academy of Sciences, Kegelgasse 27, 1030 Vienna, Austria \\ 2 Department of Materials Science and Engineering, University of Ioannina, 45110 Ioannina, Greece \\ ${ }^{3}$ Laboratory for Particle Physics, Paul Scherrer Institute, 5232 Villigen, Switzerland \\ ${ }^{4}$ LIBPhys, Physics Department, University of Coimbra, 3004-526 Coimbra, Portugal \\ 5 Zentralinstitut für Elektronik, Forschungszentrum Jülich GmbH, 52425 Jülich, Germany \\ ${ }^{6}$ Institut für Kernphysik, Forschungszentrum Jülich, 52425 Jülich, Germany \\ ${ }^{7}$ Laboratoire Kastler Brossel, Sorbonne Université, CNRS, ENS-PSL Research University, Collège de France, 4, place Jussieu, 75005 Paris, \\ France \\ ${ }^{8}$ Institut für Theoretische Physik Universität Zürich, 8057 Zürich, Switzerland \\ ${ }^{9}$ Institut des NanoSciences de Paris, CNRS, Sorbonne Université, 4 place Jussieu, 75005 Paris, France \\ ${ }^{10}$ I3N, Department of Physics, Aveiro University, 3810 Aveiro, Portugal \\ 11 Atominstitut, TU Wien, 1020 Vienna, Austria \\ 12 Bosch Termotecnologia, S.A., EN 16-Km 3,7, 3800-533 Cacia Aveiro, Portugal \\ ${ }^{13}$ Department of Radiology and Nuclear Medicine, General Hospital of the City of Vienna, 1090 Vienna, Austria \\ 14 DAHER Nuclear Technologies GmbH, 63457 Hanau, Germany \\ ${ }^{15}$ Ringkjøbing Gymnasium, Vasevej 24, 6950 Ringkjøbing, Denmark \\ 16 Physics Department, National Tsing Hua University, Hsinchu 300, Taiwan \\ 17 WTI GmbH, 52428 Jülich, Germany
}

Received: 2 October 2020 / Accepted: 4 February 2021 / Published online: 23 February 2021

(C) The Author(s) 2021

Communicated by Klaus Blaum

\begin{abstract}
The hadronic width of the ground state of pionic hydrogen has been redetermined by X-ray spectroscopy to be $\Gamma_{1 s}^{\pi \mathrm{H}}=\left(856 \pm 16_{\text {stat }} \pm 22_{\text {sys }}\right) \mathrm{meV}$. The experiment was performed at the high-intensity low-energy pion beam of the Paul Scherrer Institute by using the cyclotron trap and a highresolution Bragg spectrometer with spherically bent crystals. Coulomb de-excitation was studied in detail by comparing its influence on the line shape by measuring the three different transitions $\mathrm{K} \alpha, \mathrm{K} \beta$, and $\mathrm{K} \gamma$ at various hydrogen densities. The pion-nucleon scattering lengths and other physical quantities extracted from pionic-atom data are in good agreement with the results obtained from pion-nucleon and nucleonnucleon scattering experiments and confirm that a consistent picture is achieved for the low-energy pion-nucleon sector with respect to the expectations of chiral perturbation theory.
\end{abstract}

\footnotetext{
a e-mail: d.gotta@fz-juelich.de (corresponding author)

b e-mail: valeri.markushin@outlook.com
}

\section{Introduction}

The last decades led to a successful theoretical description of strong-interaction phenomena at low energies within the framework of an effective field theory (EFT), Chiral Perturbation Theory $(\chi \mathrm{PT})$. It exploits the chiral symmetry of the QCD Lagrangian in an ideal world of the massless light quarks $u, d$, and even $s$ (chiral limit) [1]. In the real world, the chiral symmetry is explicitly broken because of the finite quark masses.

The small current quark masses of 2,5 , and $95 \mathrm{MeV} / \mathrm{c}^{2}$ for the $u, d$, and $s$ quarks [2], however, are unable to explain the masses of the hadron multiplets. The masses of the pseudoscalar mesons require both finite current quark mass values and a non-vanishing chiral condensate [3]. However, as the current quark masses are small compared to the QCD scale corresponding to about the nucleon mass, the zero mass limit is still closely fulfilled. This especially holds true for pions exemplified by the ratio $\left(m_{\pi} / m_{p}\right)^{2} \approx 2 \% \ll 1$. Thus, $\chi$ PT allows a perturbative approach, where underlying symme- 
try properties of the QCD Lagrangian manifest themselves in observables as scattering lengths by means of low-energy theorems [1,4-7].

A chiral expansion ordered by counting the powers of (small) momenta, the quark-mass difference $\left(m_{d}-m_{u}\right)$ when restricting to $\mathrm{SU}(2)$, and the fine structure constant $\alpha$ allows one to include on the same footing strong isospin-breaking effects resulting from the $u$ and $d$ quark mass difference and those of electromagnetic origin. The unknown structure of the effective interaction at short distances arising from the QCD Lagrangian is parametrized by so-called low-energy constants (LECs), which must be taken from experiment as long as results from lattice-QCD calculations are not available.

According to its origin, $\chi \mathrm{PT}$ works best for the lightest quarks $u$ and $d$ as combined in the lightest hadron, the pion, and for the description of the pion-pion interaction [8,9] but requires a demanding experimental approach $[10,11]$. On the other hand, it has been shown that such an approach can also be successfully applied to the meson-nucleon case, then denoted as Heavy Baryon Chiral Perturbation Theory (HB $\chi \mathrm{PT})$ [12-15]. The theoretical progress in $\mathrm{HB} \chi \mathrm{PT}$ has reached already the $3 \mathrm{rd}$ and 4 th order in the chiral expansion for the scattering amplitudes [16-18].

In view of the theoretical achievements, the extraction of scattering lengths from experiment is desirable at a level of $1 \%$ or better. For such studies, hadronic atoms provide an ideal laboratory [19] because normalisation problems that are inherent to partial-wave analyses when using scattering data do not occur. The strong interaction leads to a level shift $\epsilon$ and a level broadening $\Gamma$ in atomic systems formed by negatively charged hadrons like pions and the nucleus. As atomic binding energies are in the few $\mathrm{keV}$ range, the measurement of $\epsilon$ and $\Gamma$ constitutes a scattering experiment at threshold.

The pion-nucleon scattering lengths can be extracted from the hadronic shift $\epsilon_{1 s}^{\pi \mathrm{H}}$ and broadening $\Gamma_{1 s}^{\pi \mathrm{H}}$ of the atomic ground state $1 s$ in pionic hydrogen $(\pi \mathrm{H})$ and, after applying 3-body corrections, also from the hadronic shift $\epsilon_{1 s}^{\pi \mathrm{D}}$ in pionic deuterium $(\pi \mathrm{D})$ (see Sect. 2). For recent reviews within the framework of $\chi$ PT see $[20,21]$.

Experimentally, $\epsilon_{1 s}$ and $\Gamma_{1 s}$ are accessible via the ( $n p-$ $1 s) \mathrm{X}$-ray transitions. The energy shift $\epsilon_{1 s}$ is of the order of a few $\mathrm{eV}$ and the broadening $\Gamma_{1 s} \approx 1 \mathrm{eV}$, whereas the $(n p-1 s) \mathrm{X}$-ray energies are around $3 \mathrm{keV}$ (see below and Table 2). In particular, the precise measurement of such a small line broadening requires high-resolution devices like crystal spectrometers, which in turn need strong X-ray sources. For this reason, precision results were not attainable before high-intensity and low-energy pion beams became available. First experiments of this kind achieved $\Gamma_{1 s}^{\pi \mathrm{H}}=$ $(970 \pm 112) \mathrm{meV}[22,23]$ and $(868 \pm 55) \mathrm{meV}[24,25]$, which corresponds to a precision of $12 \%$ and $7 \%$, respectively.
The result described here completes a long series of experiments [26] aiming at an improved determination of the strong-interaction effects both in pionic hydrogen and deuterium. The results for the shifts,

$\epsilon_{1 s}^{\pi \mathrm{H}}=(7.0858 \pm 0.0096) \mathrm{eV}$ and

$\epsilon_{1 s}^{\pi \mathrm{D}}=(-2.356 \pm 0.031) \mathrm{eV}$,

have been published earlier in [27-29]. In this paper, the sign convention for the shift is $\epsilon \equiv E_{\mathrm{X}}-E_{\mathrm{QED}}$, which corresponds to the change of the X-ray transition energy $E_{\mathrm{X}}$ compared to the pure electromagnetic value $E_{\mathrm{QED}}$. It is the negative of the change of total energy of the pionic atom due to the strong interaction.

In order to improve the accuracy for $\Gamma_{1 s}^{\pi \mathrm{H}}$, a specifically developed cyclotron trap was used together with a Johanntype spectrometer equipped with spherically bent Bragg crystals as well as a large area array of charge-coupled devices (CCDs), and an especially tailored concrete shielding. The experiment benefited from a 4-fold improved beam intensity provided at the Paul Scherrer Institute (Villigen, Switzerland). Statistics and peak-to-background ratio were improved by a factor of about 30 and 50, respectively, in comparison to [24,25]. In addition, a new calibration method was introduced: narrow fluorescence X-rays emitted from highly charged ions were used to determine the spectrometer resolution function with maximum possible accuracy for the given experimental approach (see Sect.4).

The main difficulty in the extraction of $\Gamma_{1 s}$ is to quantify the Doppler broadening effect on the line shape of the X-ray transitions. As discussed in Sect. 3, the kinetic energy distribution of the $\pi \mathrm{H}$ atoms changes during the atomic cascade due to collisional processes, which are density dependent. As a result, the influence of the Doppler effect depends on the initial state $n p$ and the target density. Therefore, three different ground-state transitions, $\pi \mathrm{H}(2 p-1 s), \pi \mathrm{H}(3 p-1 s)$, and $\pi \mathrm{H}(4 p-1 s)(\mathrm{K} \alpha, \mathrm{K} \beta$, and $\mathrm{K} \gamma)$, have been measured at hydrogen densities between 3.9 bar and liquid $\left(\mathrm{LH}_{2}\right)$, in order to ensure that the Doppler effect is consistently taken into account in the analysis of the line shapes.

The analysis of the spectra as described in Sect. 5 uses a model for the kinetic energy distribution which is derived from a preceding experiment with muonic hydrogen and is introduced in Sect. 5.1. It is applied both in a $\chi^{2}$ analysis (frequentist analysis Sect. 5.2) and in a Bayesian analysis (Sect. 5.3).

The use of a Bayesian approach was especially motivated by the case of pionic deuterium. There, the occurrence of a bias in a frequentist method [30], influencing the extracted values for $\Gamma_{1 s}^{\pi \mathrm{D}}$, has been studied in detail [29]. Such a bias stems from the - principally unknown - difference of the probability distribution of the data itself and the one assumed for the model. An estimate for the bias of the parameter $\Gamma_{1 s}^{\pi \mathrm{D}}$ 
was found by applying the same model as used for the analysis to a series of Monte-Carlo simulations generating the $\mathrm{X}$-ray line shape and by comparing input and output parameters. Such a lengthy procedure may be circumvented by using Bayesian methods, which are supposed to be free from bias effects in parameter estimation [31]. However, they suffer from different drawbacks, e. $g$. the influence of the choice of a prior distribution.

A comparison of the results of the two analysis methods establishes an independent consistency check (Sect. 5.4) and is in line with recent assessments recommending to use both approaches as good practice in evaluating data. Such a comparison of Bayesian and frequentist approaches has been already applied for the study of the twin-system muonic hydrogen [32,33], which completed this series of experiments. The features of the two evaluation methods are outlined in apps. A.1 and A.2 of Ref. [33].

A discussion of the different sources of systematic uncertainties refers to the influence of the different cuts in the treatment of the spectra (Sect. 5.5.1) and investigates the systematic error which is inherent to the model used for the kinetic energy distribution (Sects. 5.5.2 and 5.5.3). In Sect. 6 the result of our experiment and its implications are presented.

\section{Strong-interaction effects}

The hadronic broadening of the $\pi \mathrm{H}$ ground state results from the s-wave charge-exchange reaction $\pi^{-} p \rightarrow \pi^{0} n$ and radiative capture $\pi^{-} p \rightarrow \gamma n$. Within a non-relativistic effective field-theoretical approach, the scattering length $a_{\pi^{-}}^{\mathrm{cex}}$ associated to the charge exchange reaction is related to $\Gamma_{1 s}^{\pi \mathrm{H}}$ by $[35,36]$

$$
\begin{aligned}
\Gamma_{1 s}^{\pi \mathrm{H}}= & \frac{4 \alpha^{3} \mu_{\mathrm{H}}^{2} c^{4}}{\hbar c} q_{0}\left(1+\frac{1}{P}\right)\left(a_{\pi^{-} p}^{\mathrm{cex}}\right)^{2} \\
& \cdot\left[1-\frac{4 \alpha \mu_{\mathrm{H}} c^{2}}{\hbar c}(\ln \alpha-1) \cdot a_{\pi^{-}} p\right. \\
& \left.+\frac{2 \mu_{\mathrm{H}} c^{4}}{(\hbar c)^{2}}\left(m_{p}+m_{\pi}-m_{n}-m_{\pi^{0}}\right) \cdot a_{\pi^{0} n}^{2}+\delta_{\epsilon}^{\mathrm{vac}}\right] .
\end{aligned}
$$

Here, $\alpha$ is the fine structure constant and $m_{\pi}$ is the mass of the charged pion. $\mu_{\mathrm{H}}=m_{\pi} /\left(1+\frac{m_{\pi}}{m_{p}}\right)$ is the reduced mass of the $\pi^{-} p$ system, and $q_{0}$ is the centre-of-mass momentum of the $\pi^{0}$. The factor $\left(1+\frac{1}{P}\right)$ takes into account the fraction of radiative capture, where the Panofsky ratio for pion capture at rest was measured to be $P=\frac{\sigma\left(\pi^{-} p \rightarrow \pi^{0} n\right)}{\sigma\left(\pi^{-} p \rightarrow \gamma n\right)}=$ $1.546 \pm 0.009$ [37]. The $\pi^{0} n$ elastic scattering length $a_{\pi^{0} n}$ for the virtual intermediate state is well approximated by the isoscalar scattering length $a^{+}$(see below) [38]. The correc- tion term $\delta_{\epsilon}^{\mathrm{vac}}=0.48 \%$ accounts for the effect of the vacuum polarization on the wave function [39]. Its uncertainty is assumed to be negligibly small compared to the experimental uncertainties [20].

The scattering length $a_{\pi^{-}}$for the elastic channel $\pi^{-} p \rightarrow \pi^{-} p$ is obtained from the hadronic shift in pionic hydrogen,

$$
\begin{aligned}
\epsilon_{1 s}^{\pi \mathrm{H}}= & \frac{2 \alpha^{3} \mu_{\mathrm{H}}^{2} c^{4}}{\hbar c} a_{\pi^{-} p} \\
& \cdot\left[1-\frac{2 \alpha \mu_{\mathrm{H}} c^{2}}{\hbar c}(\ln \alpha-1) \cdot a_{\pi^{-} p}+\delta_{\epsilon}^{\mathrm{vac}}\right] .
\end{aligned}
$$

Equations (3) and (4) are modifications of the DeserGoldberger-Baumann-Thirring (DGBT) formula [40] and include corrections up to order $O\left(\alpha^{4}\right)[36,41,42]$. The most recent measurement of $\epsilon_{1 s}$ and extraction of $a_{\pi^{-}} p$ is discussed in detail in Ref. [27,28] (see also Eq. (23)).

In the limit of isospin conservation, the scattering lengths of all reactions $\pi N \rightarrow \pi N$ can be expressed by the isoscalar and isovector $s$-wave scattering lengths $a^{+}$ and $a^{-}$, which are given in terms of the elastic reactions $\pi^{-} p \rightarrow \pi^{-} p$ and $\pi^{+} p \rightarrow \pi^{+} p$ by

$a^{ \pm}=\frac{1}{2}\left(a_{\pi^{-} p} \pm a_{\pi^{+}}\right)$.

In terms of the two possible isospin combinations $I=1 / 2$ and $I=3 / 2, a^{+}$and $a^{-}$have the form

$a^{+}=\frac{1}{3}\left(a_{1 / 2}+2 a_{3 / 2}\right)$ and

$a^{-}=\frac{1}{3}\left(a_{1 / 2}-a_{3 / 2}\right)$.

The relations between the scattering lengths in the isospin conservation limit, $a^{ \pm}$, and the ones used in Eqs. (3) and (4) are given by

$a_{\pi^{-} p}^{\text {cex }}=-\sqrt{2} a^{-}+\triangle a_{\pi^{-} p}^{\text {cex }}$ and

$a_{\pi^{-} p}=\left(a^{+}+a^{-}\right)+\triangle a_{\pi^{-}} p$.

The corrections $\triangle a$ have been calculated at various levels and approaches during past decades [16,36,41-49]. Numerical values and, in particular, the uncertainties of the various $\triangle a$ were subject to significant changes resulting in nonsatisfactory conclusions on the scattering lengths $a^{+}$and $a^{-}$ in the year 1998 when this experiment was proposed.

The most recent determinations for $\triangle a$ have been worked out within the framework of $\chi \mathrm{PT}$ in next-to-leading order (NLO) $[21,38,50,51]$ :

$$
\begin{aligned}
\triangle a_{\pi^{-} p}^{\mathrm{cex}} & =(0.4 \pm 0.9) \cdot 10^{-3} m_{\pi}^{-1} \text { and } \\
\triangle a_{\pi^{-}} p & =(-8.1 \pm 2.8) \cdot 10^{-3} m_{\pi}^{-1} .
\end{aligned}
$$

These corrections constitute the isospin violating contributions of strong $\left(m_{u} \neq m_{d}\right)$ and electromagnetic origin and 
are assumed to be complete within the given order of the chiral expansion. The large errors are due to the poor knowledge of LECs appearing in the chiral expansion.

For $\pi \mathrm{D}$, the modified Deser formula has the form [52]

$$
\begin{aligned}
-\epsilon_{1 s}^{\pi \mathrm{D}}-i \frac{\Gamma_{1 s}^{\pi \mathrm{D}}}{2}= & -\frac{2 \alpha^{3} \mu_{\mathrm{D}}^{2} c^{4}}{\hbar c} a_{\pi \mathrm{D}} \\
& \cdot\left[1-\frac{2 \alpha \mu_{\mathrm{D}} c^{2}}{\hbar c}(\ln \alpha-1) \cdot a_{\pi \mathrm{D}}+\delta_{\mathrm{D}}^{\mathrm{vac}}\right],
\end{aligned}
$$

where the related pion-deuteron scattering length $a_{\pi \mathrm{D}}$ is complex. Here, $\mu_{\mathrm{D}}$ is the reduced mass of the $\pi^{-} d$ system and $\delta_{\mathrm{D}}^{\mathrm{vac}}=0.51 \%$ [39].

The real part of the scattering length $\operatorname{Re} a_{\pi \mathrm{D}}$ gives the leading contribution to $\epsilon_{1 s}^{\pi \mathrm{D}}$ and can be expressed as sum of two- and three-body terms $a_{\pi \mathrm{D}}^{(2)}$ and $a_{\pi \mathrm{D}}^{(3)}$

$\operatorname{Re} a_{\pi \mathrm{D}}=a_{\pi \mathrm{D}}^{(2)}+a_{\pi \mathrm{D}}^{(3)}$

The two-body term $a_{\pi \mathrm{D}}^{(2)}=a_{\pi^{-} p \rightarrow \pi^{-} p}+a_{\pi^{-} n \rightarrow \pi^{-} n}$ is essentially $2 \cdot a^{+}$and, thus, provides an important constraint on the results for $a^{+}$and $a^{-}$as obtained from $\epsilon_{1 s}^{\pi \mathrm{H}}$ and $\Gamma_{1 s}^{\pi \mathrm{H}}$. Though $a_{\pi \mathrm{D}}^{(2)}$ involves substantial isospin-violating corrections and $a_{\pi \mathrm{D}}^{(3)}$ depends in leading order on $\left(a^{-}\right)^{2}$, the corrections are well under control. The theoretical framework is introduced and discussed in detail in Refs. [20,38,51-58].

The imaginary part $\operatorname{Im} a_{\pi \mathrm{D}}$, which gives the leading contribution to $\Gamma_{1 s}^{\pi \mathrm{D}}$, measures the transition strength of $s$ wave pions on an isoscalar nucleon-nucleon pair $\pi N N \leftrightarrow$ $N N$ [59] and is an independent quantity not related to the scattering lengths $a^{+}$and $a^{-}[29,60,61]$.

The following relations hold in the isospin conservation limit:

$a_{\pi^{-} p}-a_{\pi^{+} p}=-\sqrt{2} a_{\pi^{-} p}^{\mathrm{cex}}=2 a^{-}$.

The deviation from the identity Eq. (14) quantifies the isospin-breaking effects. In terms of measurable quantities, it can be described by the ratio

$R=2 \frac{a_{\pi^{+} p}-a_{\pi^{-} p}-\sqrt{2} a_{\pi^{-} p}^{\mathrm{cex}}}{a_{\pi^{+} p}-a_{\pi^{-} p}+\sqrt{2} a_{\pi^{-} p}^{\mathrm{cex}}}$,

and is expected to be of the order of a few \% [38].

The isovector scattering length $a^{-}$and correspondingly $\Gamma_{1 s}^{\pi \mathrm{H}}$ are connected to other important quantities. These include the pion-nucleon coupling constant, the Goldberger-Treiman discrepancy, and the pion-production amplitude $\gamma n \rightarrow \pi^{-} p$ at threshold.
The Goldberger-Miyazawa-Oehme (GMO) sum rule [62], which can be written in the form [51,63]

$$
\begin{aligned}
\frac{g_{c}^{2}}{4 \pi}= & \left(\left(\frac{m_{p}+m_{n}}{m_{\pi}}\right)^{2}-1\right) \\
& \cdot\left[\left(1+\frac{m_{\pi}}{m_{p}}\right) \frac{m_{\pi}}{4} \cdot\left(a_{\pi^{-} p}-a_{\pi^{+} p}\right)-\frac{m_{\pi}^{2}}{2} \cdot J\right],
\end{aligned}
$$

relates the scattering lengths $a_{\pi^{-}}$and $a_{\pi^{+}}$to the charged pion-nucleon coupling constant $g_{c}^{2} / 4 \pi$. The GMO sum rule essentially constitutes a forward dispersion relation containing the integral $J$ ranging over all pion momenta which, employing the optical theorem, can be expressed as the difference $\sigma_{\pi^{-} p}^{\text {tot }}-\sigma_{\pi^{+} p}^{\text {tot }}$ of the Coulomb-corrected total cross sections. After applying appropriate corrections (see Eqs. (25) and (30)), the scattering length difference $a_{\pi^{-} p}-a_{\pi^{+} p}$ can be related to $a_{\pi^{-} p}$ and $a_{\pi^{-}}^{\mathrm{cex}}$, i.e. to the hadronic shift $\epsilon_{1 s}^{\pi \mathrm{H}}$ and to the level broadening $\Gamma_{1 s}^{\pi \mathrm{H}}$ in pionic hydrogen [51,63,64].

In the chiral limit, the Goldberger-Treiman relation $m_{N} g_{A}=f_{\pi} g_{c}$ holds true [65], where $g_{A}$ is the axial-vector coupling constant in $\beta$-decay, $f_{\pi}$ is the pion decay constant, and the average nucleon mass $m_{N}=\frac{m_{p}+m_{n}}{2}$. The deviation from the Goldberger-Treiman relation, the GoldbergerTreiman discrepancy [66], can be written as

$$
\triangle G T=1-\frac{m_{N} g_{A}}{f_{\pi} g_{c}}
$$

with the pion-nucleon coupling constant $g_{c}$ obtained, e.g. from the GMO sum rule. It measures the explicit chiral symmetry breaking by the quark masses and is expected to be small in the standard chiral symmetry breaking $(\approx 1.5 \%)$ because of the small $u$ and $d$ quark masses [50,67-69].

Furthermore, the Kroll-Ruderman theorem connects $g_{c}$ with s-wave pion photoproduction $\gamma N \rightarrow \pi N$ [70]. For $\pi^{-}$ production, the threshold electric dipole amplitude reads

$E_{0+}^{\mathrm{thr}}\left(\pi^{-} p\right)=\frac{\sqrt{4 \pi \alpha} g_{c}}{4 \pi \sqrt{2} m_{\pi}\left(1+\frac{m_{\pi}}{m_{p}}\right)}+$ h.o.

Here, the chiral corrections (h.o.) to the leading-order term have been calculated to third order in the framework of $\chi P T$ [71-73].

The isoscalar $\pi N$ amplitude $a^{+}$is related the $\pi N \sigma$ term $\sigma_{\pi N}$ [74], which encodes the contribution of the lightest quark doublet to the masses of the nucleons. Furthermore, its connection to the scalar coupling of dark matter [75-77] or $\mu \rightarrow e$ lepton flavor violation $[78,79]$ is of interest in the context of new physics research.

The long-standing debate about the extraction of $\sigma_{\pi N}$ is, among other reasons, due to the difficulties arising from subthreshold extrapolation of amplitudes [80,81]. In the mean- 
time, substantial progress has been made in the development of concepts for such a procedure. Within the framework of dispersion relations, a direct correlation including chiral corrections between $\sigma_{\pi N}$ and the $\pi N$ s-wave scattering lengths has been established [21,82].

Most analyses of recent pionic-atom [83] and low-energy scattering data lead to $\sigma_{\pi N} \approx 60 \mathrm{MeV}[84,85]$. Selection of one particular data set $\left(\sigma_{\pi N}=(44 \pm 12) \mathrm{MeV}[86]\right)$ or the choice of a particular partial-wave analysis [87] lead to non-decisive or scheme-dependent results. In contrast, lattice calculations [88-91] typically yield such small values being in tension by $3-4 \sigma$ with most data-based results [92]. One possibility to support solutions of this puzzle, is to confirm the reliability of experimental information, $e . g$. the pionatom data base.

\section{Pionic hydrogen atomic cascade}

After slowing down to a few $\mathrm{eV}$, a heavy negatively charged particle $\left(x^{-}=\mu^{-}, \pi^{-}, K^{-}\right.$, or $\left.\bar{p}\right)$, while passing through the atomic or molecular electron cloud and losing further its kinetic energy, is eventually captured in a highly excited bound state where an atomic quantum cascade starts. These initial states are comparable in size with the hydrogen atom and have the principle quantum number $n_{c} \approx \sqrt{\mu_{x} / m_{e}}$, where $\mu_{x}$ denotes the $x^{-} p$ reduced mass $[93,94]$. In the case of pionic hydrogen, $n_{c} \approx 16$. The initial distributions in $n_{c}$ and the angular momentum quantum number $\ell$ have been calculated to be broad distributions [95-98].

Before the pions undergo the radiative transitions $(n p \rightarrow 1 s)$ that can be observed experimentally, different collisional processes occur in the atomic cascade (Fig. 1). While these collisional processes are not directly observable, they change the kinetic energy of the $\pi \mathrm{H}$ atom and, therefore, influence the line shape of the measured radiative transitions due to Doppler broadening. For this reason, a good understanding of the atomic cascade is crucial to a proper evaluation of the measured data.

Traditionally, the collisional processes $\left(\pi^{-} p\right)_{n l}+\mathrm{H} \rightarrow$ $\left(\pi^{-} p\right)_{n^{\prime} l^{\prime}}+\mathrm{H}$ were classified according to their main effect in the atomic cascade (Table 1). In particular, during the collisions Stark mixing causes transitions with $n=n^{\prime}$ and $l^{\prime} \neq l$ that can produce $n s$ states where nuclear absorption takes place with high probability [93]. The nuclear absorption via the Stark mixing significantly reduces the intensities of the radiative transitions, especially at high hydrogen density.

The Coulomb transitions [99,100] correspond to the collisions with $n^{\prime}<n$ where the transition energy is shared between colliding particles, which have comparable masses, thus resulting in a significant acceleration of the $\pi \mathrm{H}$ system. Elastic scattering, where the principal quantum number remains unchanged $\left(n^{\prime}=n\right)$, plays an important role in the

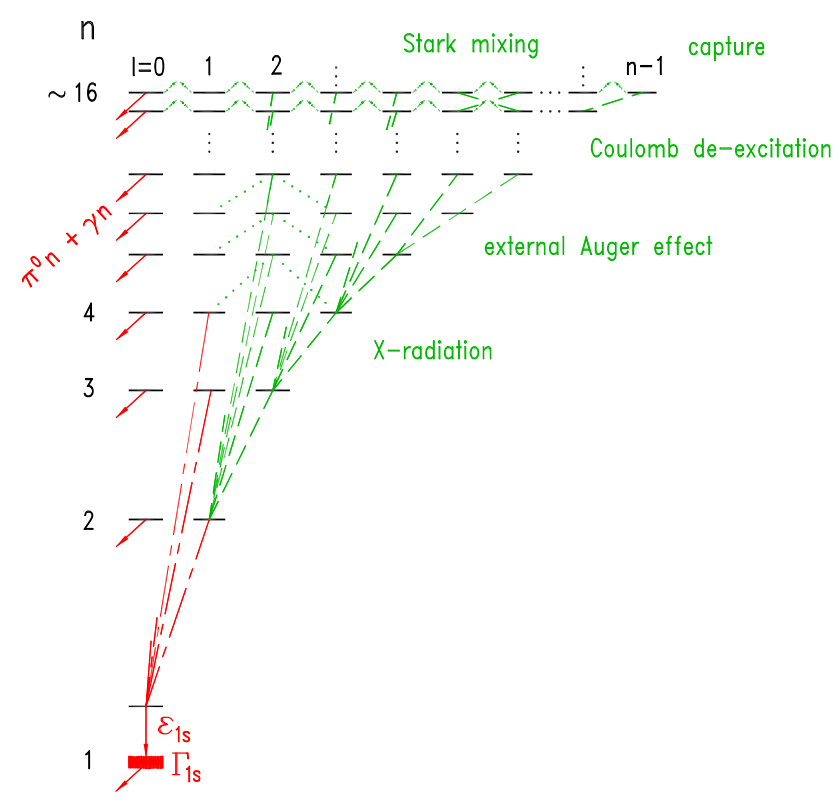

Fig. 1 Atomic de-excitation cascade after pion capture in hydrogen. In this experiment, the 3 most intense $\mathrm{X}$-ray lines $\pi \mathrm{H}(2 p-1 s), \pi \mathrm{H}(3 p-$ $1 s)$, and $\pi \mathrm{H}(4 p-1 s)$ have been studied. Because strong-interaction effects in the $n p$ states are negligibly small and radiative widths $\Gamma_{\mathrm{X}} \ll$ $\Gamma_{1 s}$ (see Table 2), the natural line width is practically equal to the $1 s$ level broadening $\Gamma_{1 s}$ due to the reactions $\left(\pi^{-} p\right)_{1 s} \rightarrow \pi^{0}+n$ and $\gamma+n$. The level shift $\epsilon_{1 s}$ is caused in leading order by elastic scattering $\pi^{-} p \rightarrow \pi^{-} p$ with the $\pi^{-} \mathrm{H}$ system being in the atomic ground state

deceleration of the pionic hydrogen, since the kinetic energy of $\left(\pi^{-} p\right)_{n l}$ is usually much higher than the kinetic energy of the $\mathrm{H}_{2}$ molecules.

The hydrogen molecular structure can be important for certain collision channels, especially at high $n$ when the size of $\left(\pi^{-} p\right)_{n l}$ is not small in comparison with the one of the hydrogen atom and when the degrees of freedom of $\mathrm{H}_{2}$ molecules play a role. In the earlier cascade calculations, different approximations were used to model the above mentioned collisional processes $[93,101]$. The modern calculation of the collisional rates treats them as different final states of the same scattering problem by using a unified framework that improves significantly the accuracy of the theoretical results [102].

The collisional processes dominate the upper and the intermediate part of the atomic cascade, because even at modest densities, the corresponding reaction rates exceed the radiative de-excitation rates. Only for small $n$, X-ray emission starts to compete and finally dominates in the very last steps of the cascade.

The rates of collisional processes scale with hydrogen density $\phi$. As a result, the cascade time rapidly decreases with the increase of the target density. The cascade times are calculated to be between $10^{-9}$ to $10^{-11} \mathrm{~s}$ in the range $\phi=10^{-3}$ to 1 [103], where $\phi=1$ corresponds to the density of liquid hydrogen. Therefore, almost all pions reach an atomic $n s$ 
Table 1 Processes contributing to the de-excitation cascade in pionic hydrogen

\begin{tabular}{ll}
\hline Process & \\
\hline Radiative transitions & $\left(\pi^{-} \mathrm{p}\right)_{n l} \rightarrow\left(\pi^{-} \mathrm{p}\right)_{n^{\prime}, l \pm 1}+\gamma$ \\
Stark transitions & $\left(\pi^{-} \mathrm{p}\right)_{n l}+\mathrm{H}_{2} \rightarrow\left(\pi^{-} \mathrm{p}\right)_{n l^{\prime}}+\mathrm{H}_{2}^{*}$ \\
External auger effect & $\left(\pi^{-} \mathrm{p}\right)_{n l}+\mathrm{H} \rightarrow\left(\pi^{-} \mathrm{p}\right)_{n^{\prime} l^{\prime}}+\mathrm{p}+\mathrm{e}^{-}$ \\
Coulomb de-excitation & $\left(\pi^{-} \mathrm{p}\right)_{n l}+\mathrm{H}_{2} \rightarrow\left(\pi^{-} \mathrm{p}\right)_{n^{\prime} l^{\prime}}+\mathrm{H}+\mathrm{H}$ \\
Elastic scattering & $\left(\pi^{-} \mathrm{p}\right)_{n l}+\mathrm{H}_{2} \rightarrow\left(\pi^{-} \mathrm{p}\right)_{n l}+\mathrm{H}_{2}^{*}$ \\
Molecular formation & $\left(\pi^{-} \mathrm{p}\right)_{n l}+\mathrm{H}_{2} \rightarrow\left(\left\{\pi^{-} \mathrm{pp}\right\}^{*} \mathrm{pee}\right)^{*}$ \\
Nuclear reaction & $\left(\pi^{-} \mathrm{p}\right)_{n s} \rightarrow \pi^{0}+\mathrm{n}, \gamma+\mathrm{n}$ \\
Pion decay & $\pi^{-} \rightarrow \mu^{-}+\overline{v_{\mu}}\left(\tau_{\pi^{-}}=26 \mathrm{~ns}\right)$ \\
\hline
\end{tabular}

The nuclear reaction channels and the pion decay terminate the atomic cascade

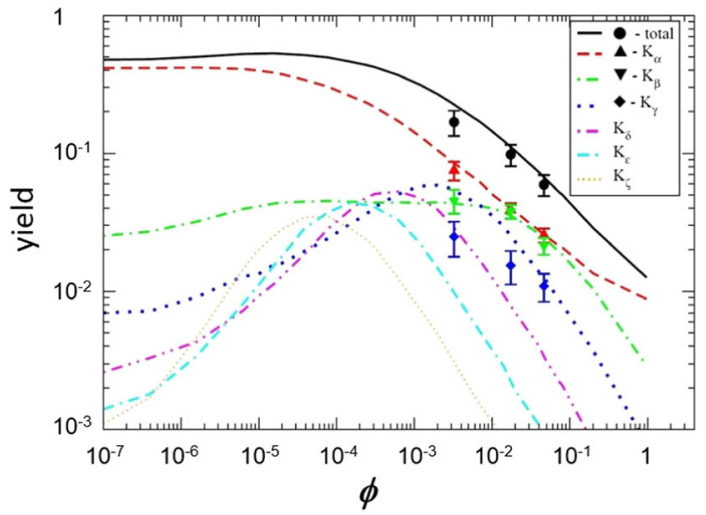

Fig. 2 Absolute K X-ray yields in pionic hydrogen vs. target density $\phi$ (normalized to liquid hydrogen density). The theoretical yields are obtained from a calculation within the framework of ESCM [106]. The experimental data (points with error bars) are from [107]

state, where the pion wave function has a large overlap with the nucleus, and the nuclear reactions of charge exchange and radiative capture occur on the femtosecond scale. Hence, the pions reaching $s$-states disappear from the de-excitation cascade in contrast to the muonic hydrogen case where muon capture from excited atomic states is negligibly small. Consequently, the X-ray yields in pionic hydrogen are about one order of magnitude smaller than the ones in muonic hydrogen. Absolute yields are in the few \% range for the densities covered by this experiment (Fig. 2).

The competition between the acceleration (Coulomb deexcitation) and the deceleration (elastic and inelastic scattering) leads to the evolution of the kinetic energy distribution during the atomic cascade, and the corresponding energy distributions can be studied experimentally for several particular states. Experimentally, the Doppler broadening was observed for the first time in the time-of-flight distribution of neutrons produced in the charge-exchange reaction $\pi^{-} p \rightarrow \pi^{0} n$ after pion capture at rest $[104,105]$ and studied later in detail in connection with a precision determination of the $\pi^{-}-\pi^{0}$ mass difference [108]. Predominantly, Coulomb de-excitation involves one atom of the $\mathrm{H}_{2}$ molecule, i.e. the collisions $\left(\pi^{-} \mathrm{p}\right)_{n l}+\mathrm{H}$ must be considered [109-111]. Therefore, the $\pi \mathrm{H}$ atom gains about one half of the de-excitation energy.

The energy gain of the $\pi \mathrm{H}$ system in the $\Delta n=1$ Coulomb transitions $(6-5),(5-4),(4-3)$, and $(3-2)$ is 18 , 34,73 , and $209 \mathrm{eV}$, respectively. For $\Delta n>1$ transitions, the energy gain is given by summing up the corresponding $\Delta n=1$ contributions. The energy acquired in the Coulomb de-excitation transition $(n+1) \rightarrow n$ accounts for a significant Doppler broadening in the subsequent X-ray line $(n p \rightarrow 1 s)$ of $\Delta E_{X^{4 p-1 s}}=1.5 \mathrm{eV}, \Delta E_{X^{3 p-1 s}}=2.1 \mathrm{eV}$, and $\Delta E_{X^{2 p-1 s}}=$ $2.9 \mathrm{eV}$, respectively. The energies of the transitions $\pi \mathrm{H}(4 p-$ $1 s), \pi \mathrm{H}(3 p-1 s)$, and $\pi \mathrm{H}(2 p-1 s)$ are about 3.0, 2.9, and $2.4 \mathrm{keV}$ (Table 2).

In contrast to Coulomb de-excitation, the kinetic energy transfer to the $\pi \mathrm{H}$ atom during the initial capture and from external Auger effect is negligibly small, because the energy difference is carried by the emitted electron. Similarly, the recoil effects from X-ray emission, the Stark collisions (at small scattering angles), and molecular formation need not be considered for a noticeable contribution to Doppler broadening. Furthermore, radiative decay from molecular states causing a line broadening due to satellite transitions is expected to be negligibly small [112-115]. No evidence for such transitions could be identified from the study of the density dependence of the X-ray energy [27,33].

At densities of $\phi \approx 10^{-2}$, also the contributions of induced Stark width, collisional broadening, and thermal motion add up at most to a negligible value of $\approx 3 \mathrm{meV} \mathrm{[33,}$ 109]. At $\phi=1$, an upper limit of $30 \mathrm{meV}$ is assumed for the induced Stark width [109].

Elastic and inelastic scattering are the only efficient deceleration processes, which compete with the acceleration caused by Coulomb de-excitation. This leads to a complex kinetic energy distribution at the time of the $\mathrm{X}$ ray emission (Fig.3). Consequently, the state-dependent development of the kinetic energy is essential for modern cascade calculations (extended standard cascade model (ESCM)) [101, 106, 116-119].

\section{Experiment}

\subsection{Set-up}

The experiment was performed in the $\pi \mathrm{E} 5$ area at the meson factory of the Paul Scherrer Institute (Fig. 4). The X-ray source has been provided by the cyclotron trap. A weakly focussing magnetic field, produced by a superconducting split-coil magnet, guided the pion beam to the centre of the trap $[120,121]$. In the centre was installed a thin-walled Kap- 

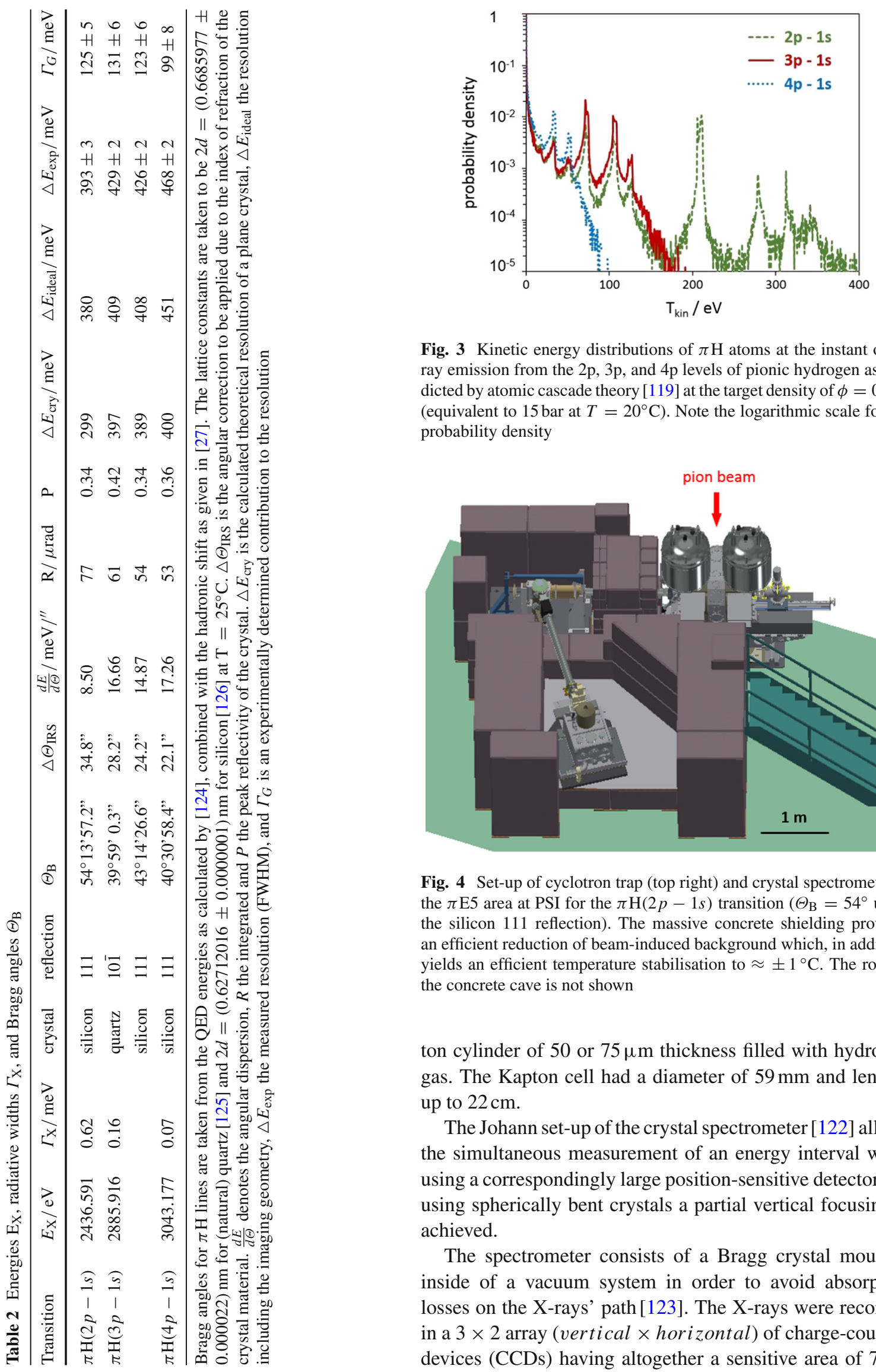

Fig. 3 Kinetic energy distributions of $\pi \mathrm{H}$ atoms at the instant of $\mathrm{X}$ ray emission from the $2 p, 3 p$, and $4 p$ levels of pionic hydrogen as predicted by atomic cascade theory [119] at the target density of $\phi=0.018$ (equivalent to $15 \mathrm{bar}$ at $T=20^{\circ} \mathrm{C}$ ). Note the logarithmic scale for the probability density

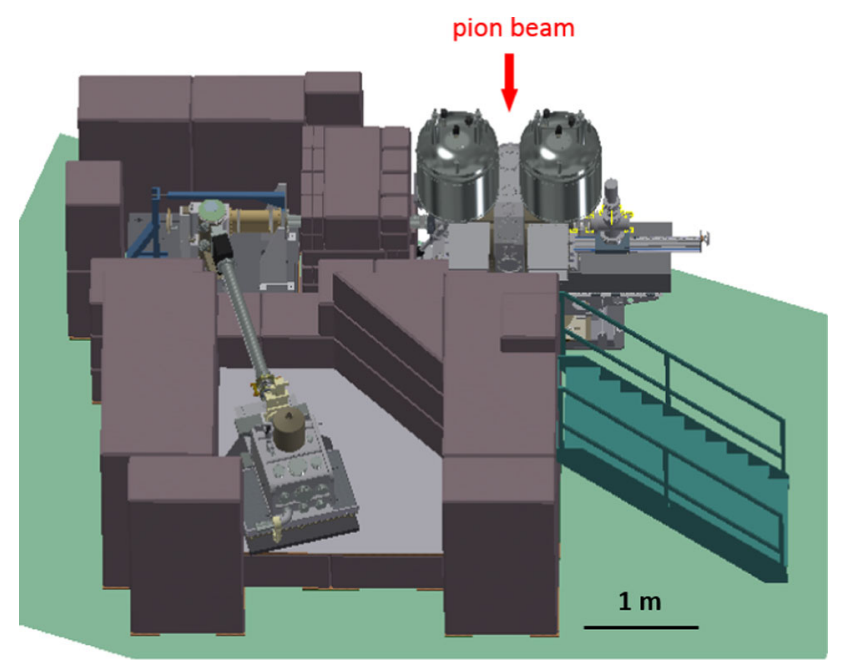

Fig. 4 Set-up of cyclotron trap (top right) and crystal spectrometer in the $\pi \mathrm{E} 5$ area at PSI for the $\pi \mathrm{H}(2 p-1 s)$ transition $\left(\Theta_{\mathrm{B}}=54^{\circ}\right.$ using the silicon 111 reflection). The massive concrete shielding provides an efficient reduction of beam-induced background which, in addition, yields an efficient temperature stabilisation to $\approx \pm 1{ }^{\circ} \mathrm{C}$. The roof of the concrete cave is not shown

ton cylinder of 50 or $75 \mu \mathrm{m}$ thickness filled with hydrogen gas. The Kapton cell had a diameter of $59 \mathrm{~mm}$ and lengths up to $22 \mathrm{~cm}$.

The Johann set-up of the crystal spectrometer [122] allows the simultaneous measurement of an energy interval when using a correspondingly large position-sensitive detector. By using spherically bent crystals a partial vertical focusing is achieved.

The spectrometer consists of a Bragg crystal mounted inside of a vacuum system in order to avoid absorption losses on the X-rays' path [123]. The X-rays were recorded in a $3 \times 2$ array (vertical $\times$ horizontal) of charge-coupled devices (CCDs) having altogether a sensitive area of $72 \times$ 
$48 \mathrm{~mm}^{2}[127,128]$. The image area of each of the six CCDs contains $610 \times 602=367220$ pixels of $40 \times 40 \mu \mathrm{m}^{2}$.

The detector plane is oriented perpendicular to the line crystal centre to CCD array centre. The focussing condition (distance crystal-detector) is given by $R_{c} \cdot \sin \Theta_{\mathrm{B}}$, where $\Theta_{\mathrm{B}}$ is the Bragg angle. The dispersion in the detector plane reads $\frac{d E}{d x}=\frac{d E}{d \Theta} \cdot \frac{1}{R_{c} \cdot \sin \Theta_{\mathrm{B}}}($ Table 2$)$.

In order to keep X-ray absorption small, the exit window of the target cell was made from a $7.5 \mu \mathrm{m}$ thick Kapton foil. For a diameter of about $50 \mathrm{~mm}$, adapted to the detector size in the direction of dispersion, such a window safely allows a pressure difference of up to about 1 bar only. Therefore, the hydrogen density was adjusted by temperature variation by means of a cooling finger. In this way, a density range from 4 bar equivalent (at $\mathrm{T}=20^{\circ} \mathrm{C}$ ) to liquid (corresponding to 787 bar) was covered (Table 3 ). A minimum density equivalent to 10 bar is necessary to stop a sufficiently large fraction of pions inside the target cell in order to achieve the statistics needed for the precise determination of the line shape.

The experimental approach is identical to the one used for the most recent determinations of the hadronic shift in pionic hydrogen, the strong-interaction effects in pionic deuterium, and for the line-shape measurement of muonic hydrogen. Accelerator conditions, experiment set-up and measurement procedure are described in more detail in the corresponding publications [27, 29,33].

\subsection{Spectrometer resolution}

For the X-ray diffraction, only silicon and quartz crystals provide the energy resolution needed in the considered energy range. In this experiment, one quartz and one silicon crystal were used cut along the (10 $\overline{1})$ and (111) plane, respectively. Crystal disks of 0.2 and $0.3 \mathrm{~mm}$ thickness and of $100 \mathrm{~mm}$ in diameter were spherically bent to radii measured to be $R_{c}=(2980.6 \pm 0.4)$ and $(2982.2 \pm 0.3) \mathrm{mm}$, respectively [129]. The area of the disks was limited twofold: (i) by a spherical aperture to a diameter of $95 \mathrm{~mm}$ in order to avoid edge effects and (ii) to $\pm 30 \mathrm{~mm}$ in the direction of dispersion to keep Johann broadening small [130].

The measured total line shape is composed of contributions from the spectrometer response, Doppler broadening due to Coulomb de-excitation, and the natural line width of the X-ray transition. As both the contributions from Doppler broadening and the natural line width are to be determined, the knowledge of the spectrometer response is essential to a level of accuracy that its influence on the results for $\Gamma_{1 s}$ remains negligible. The spectrometer response itself is composed of the rocking curve reflecting the intrinsic resolution of the crystal material, the imaging properties resulting from the bent-crystal set-up, and possible imperfections from setup geometry and crystal mounting.
The spectrometer response was determined by using Xrays emitted from highly-charged ions (Fig.5). The ions were produced by means of discharge in a dedicated electron-cyclotron ion resonance trap (ECRIT) installed in the centre of the cyclotron trap [131]. In this way, the count rates up to 10000 per hour could be achieved for calibration lines, which is necessary for a precise mapping of the reflection properties and, in particular, the tails of the response function. The resolution was determined under conditions as close as possible to the $\pi \mathrm{H}$ experiment. The crystal spectrometer was directly attached to the cyclotron trap with the embedded ECRIT in the same geometrical set-up as realized for the experiment [132]. The plasma cloud provided an extended X-ray source similar to the dimensions of the hydrogen target cell.

The M1 X-ray lines from helium-like argon, chlorine, and sulphur have energies of 3104,2765 , and $2430 \mathrm{eV}$, respectively, which are very close to the energies of the three $\pi \mathrm{H}$ lines measured in this experiment (Table 2). M1 transitions $1 s 2 s^{3} S_{1} \rightarrow 1 s^{2}{ }^{1} S_{0}$ originate from metastable initial states and have natural line widths of about $10 \mathrm{neV}$ [133]. The Doppler effect on the transitions has been measured to be 15-80 meV for Ar, which corresponds to a kinetic energy of the ions of $1-30 \mathrm{eV}$ [134-136]. Such a Doppler broadening contributes at maximum with a few meV to the spectrometer resolution (Table 2). Therefore, the measured line shape is taken as the direct response of the apparatus.

As a main result, the crystal response function has been measured for the three different energies with an accuracy of better than $1 \%$ in terms of FWHM. In particular, the precise knowledge of the asymmetric line shape assures that the contribution of spectrometer response to the uncertainty of $\Gamma_{1 s}$ is negligibly small within the statistics achieved for the $\pi \mathrm{H}$ spectra.

The measurements were compared to a modelling of the resolution and found to be close to the theoretical limit. Here, the resolution function at a given energy was constructed from the rocking curve as calculated from the dynamical theory of diffraction for a perfect flat crystal (Fig. 6) convoluted with the imaging properties of the whole experimental set-up by means of a Monte-Carlo ray-tracing code. The result constitutes the ideal response with a corresponding line width $\triangle E_{\text {ideal }}$ (FWHM) for a given set-up [123]. The intrinsic resolution (rocking curve) was calculated with the code XOP $[139,140]$.

Evaluation of the results showed that the line shape measured with the ECRIT set-up is already very close to the ideal response. It is sufficient to introduce an additional Gaussian contribution $\Gamma_{G}$ to take into account imperfections of the crystal material and mounting for a good description of the line shape. The values for $\Gamma_{G}$ were found from fits to the measured spectra of the sulphur, chlorine, and argon M1 transitions $[137,138]$. The corresponding values and uncertainties 
Table 3 Experimental conditions for the various measurements of $\pi \mathrm{H}$ transitions (label A1 - C2)

\begin{tabular}{|c|c|c|c|c|c|c|c|c|}
\hline \multirow[t]{3}{*}{ Label } & \multirow[t]{3}{*}{ Transition } & \multirow[t]{3}{*}{ Reflection } & \multicolumn{2}{|l|}{$\mathrm{H}_{2}$ density } & \multirow{3}{*}{$\begin{array}{l}\text { Target } \\
\text { Temperature } \\
\text { / K }\end{array}$} & \multicolumn{2}{|c|}{ Counts $\pi \mathrm{H}$} & \multirow{3}{*}{$\begin{array}{l}\text { Background } \\
\text { / CCD channel }\end{array}$} \\
\hline & & & $\underline{p_{e q}}$ & $\phi$ & & & & \\
\hline & & & \multicolumn{2}{|l|}{ / bar } & & / hour & /per transition & \\
\hline A1 & $\pi \mathrm{H}(2 p-1 s)$ & Si 111 & $10.1 \pm 0.3$ & $0.0121 \pm 0.0003$ & $37.7 \pm 0.5$ & $44 \pm 1$ & $6867 \pm 102$ & $1.98 \pm 0.12$ \\
\hline A2 & $\pi \mathrm{H}(2 p-1 s)$ & Si 111 & $11.0 \pm 0.5$ & $0.0142 \pm 0.0006$ & $23.3 \pm 0.5$ & $94 \pm 1$ & $45611 \pm 257$ & $9.45 \pm 0.31$ \\
\hline B1 & $\pi \mathrm{H}(3 p-1 s)$ & qu $10 \overline{1}$ & $3.9 \pm 0.3$ & $0.0042 \pm 0.0003$ & $85 \pm 1$ & $17 \pm 1$ & $1004 \pm 38$ & $1.18 \pm 0.06$ \\
\hline B2 & $\pi \mathrm{H}(3 p-1 s)$ & Si 111 & $9.5 \pm 0.3$ & $0.0114 \pm 0.0003$ & $37.5 \pm 0.5$ & $43 \pm 1$ & $7220 \pm 96$ & $3.35 \pm 0.11$ \\
\hline B3 & $\pi \mathrm{H}(3 p-1 s)$ & qu $10 \overline{1}$ & $27.9 \pm 0.6$ & $0.0378 \pm 0.0003$ & $20.2 \pm 0.5$ & $28 \pm 1$ & $6988 \pm 93$ & $3.39 \pm 0.11$ \\
\hline B4 & $\pi \mathrm{H}(3 p-1 s)$ & qu $10 \overline{1}$ & 787 & 1 & $16.9 \pm 0.3$ & $16 \pm 1$ & $1749 \pm 49$ & $1.83 \pm 0.07$ \\
\hline $\mathrm{C} 1$ & $\pi \mathrm{H}(4 p-1 s)$ & Si 111 & $9.6 \pm 0.3$ & $0.0115 \pm 0.0003$ & $37.7 \pm 0.5$ & $24 \pm 1$ & $5648 \pm 89$ & $6.86 \pm 0.15$ \\
\hline $\mathrm{C} 2$ & $\pi \mathrm{H}(4 p-1 s)$ & Si 111 & $9.2 \pm 0.5$ & $0.0118 \pm 0.0003$ & $26.4 \pm 0.3$ & $25 \pm 1$ & $4637 \pm 79$ & $3.17 \pm 0.11$ \\
\hline
\end{tabular}

The $\mathrm{H}_{2}$ target densities are given as equivalent pressure $p_{e q}$ (in bar corresponding to a temperature of $20^{\circ} \mathrm{C}(\mathrm{NTP})$ ) and as fraction $\phi$ of liquid hydrogen density. Counts are results of fits to the line shape. The accelerator current varied between 1.2 and $2 \mathrm{~mA}$, which corresponds to pion fluxes arriving in the $\pi \mathrm{E} 5$ area of about $(3-5) \cdot 10^{9} \pi^{-} / \mathrm{s}$
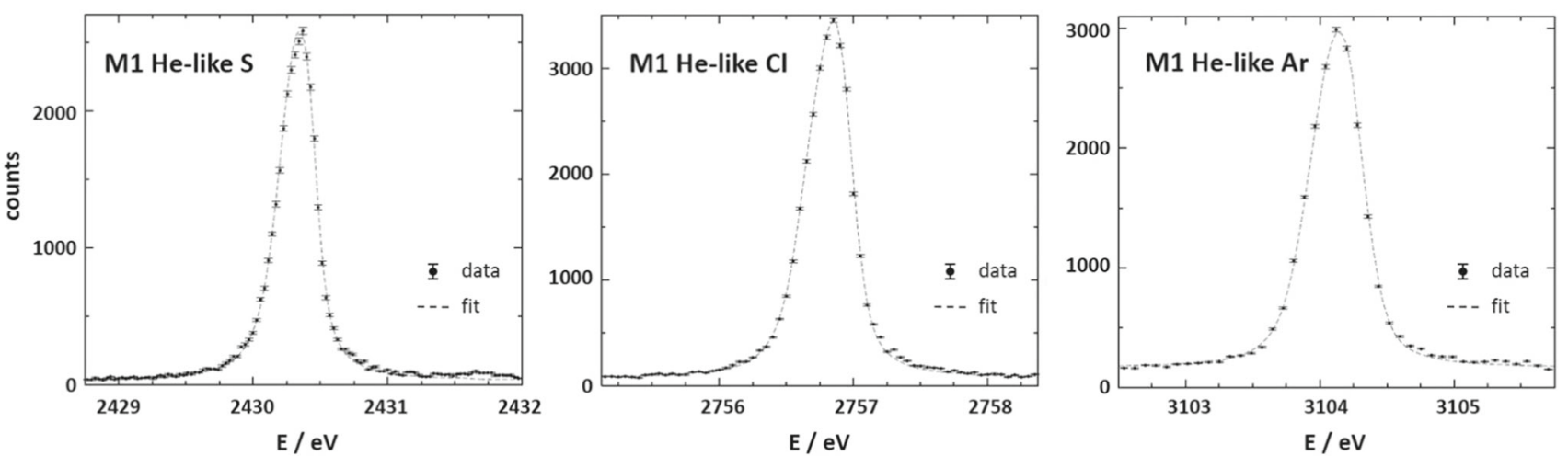

Fig. 5 The measured response functions of the spectrometer equipped with a Si(111) crystal by using M1 X-ray lines from helium-like argon, chlorine, and sulphur. For details see refs. [137,138]
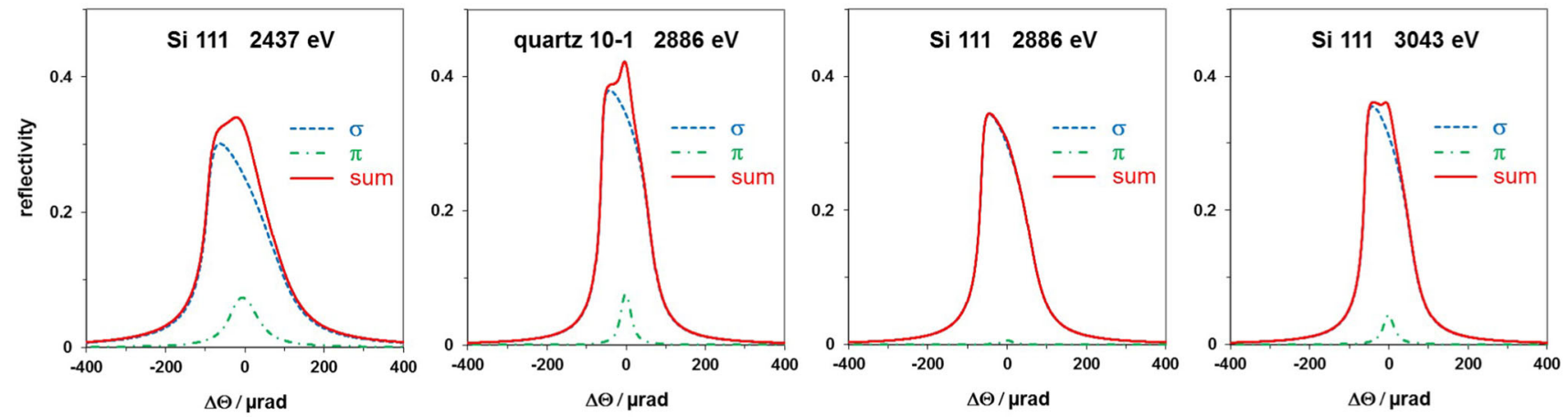

Fig. 6 The flat crystal response for the quartz $10 \overline{1}$ and Si 111 reflections for the energies of the $\pi \mathrm{H}(2-1), \pi \mathrm{H}(3-1)$, and $\pi \mathrm{H}(4-1)$ transitions as calculated with the code XOP $[139,140] .1 \mu \mathrm{rad}$ corresponds to 0.06 " (seconds of arc) 

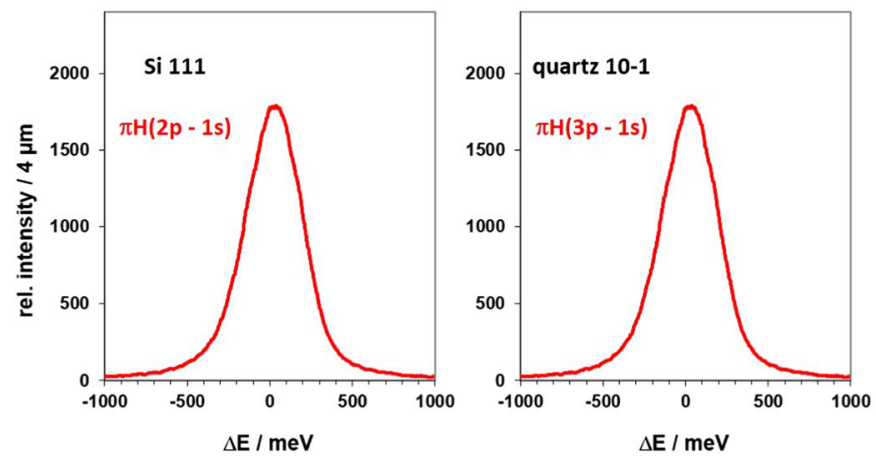

Fig. 7 The spectrometer response including the imaging properties of the set-ups for the quartz $10 \overline{1}$ and $\mathrm{Si} 111$ reflections for the energies corresponding to the $\pi \mathrm{H}(2-1), \pi \mathrm{H}(3-1)$, and $\pi \mathrm{H}(4-1)$ transitions.

of the total width $\triangle E_{\exp }$ are given in Table 2. The response functions including the Gaussian contribution are shown in Fig. 7.

As described in Refs. [137,138,141], the data taken with the ECRIT set-up were also used to determine precisely the crystal's bending radii and resolutions for various apertures, to identify possible miscuts and their orientations, as well as to validate the X-ray tracking code which includes the aberrations to all orders for the assumed geometry [123].

\subsection{Data processing}

The Bragg reflection of a mono-energetic X-ray line generates a cone-section like hit pattern in the detector plane. The position resolution is given by the granularity of the CCD array. The low-energetic X-rays create charge only in one or two adjacent pixels, because of the small photo-electron range of a few $\mu \mathrm{m}$. A substantial background reduction has been achieved by a pattern analysis followed by the rejection of larger clusters stemming from beam-induced background, which has been further improved by applying an appropriate window in the pixel charge spectrum (Fig. 8).

The detector resolution in terms of the collected charge has been determined by a Gaussian fit to the various $\pi \mathrm{H}$ peaks and found to be between 120 and $300 \mathrm{eV}$ (FWHM) for the individual CCDs. Of the events attributed to the $\pi \mathrm{H}$ lines, about $75 \%$ are single and about $25 \%$ two-pixel clusters. Defect (horizontal) CCD lines ( $0-4$ per CCD) and additional individual hot pixels $(\approx 10$ per $\mathrm{CCD})$ were masked by software.

The curvature of the reflection has been corrected by means of a parabolic fit before projecting onto the axis of dispersion (Fig. 9). The projection constitutes the energy spectra to be analysed for the Doppler and the hadronic broadening (Fig. 10). The uncertainty of the curvature correction influences the line width by less than 0.1 pixels, which corresponds to $3-7 \mathrm{meV}$ for the various transitions. Such an
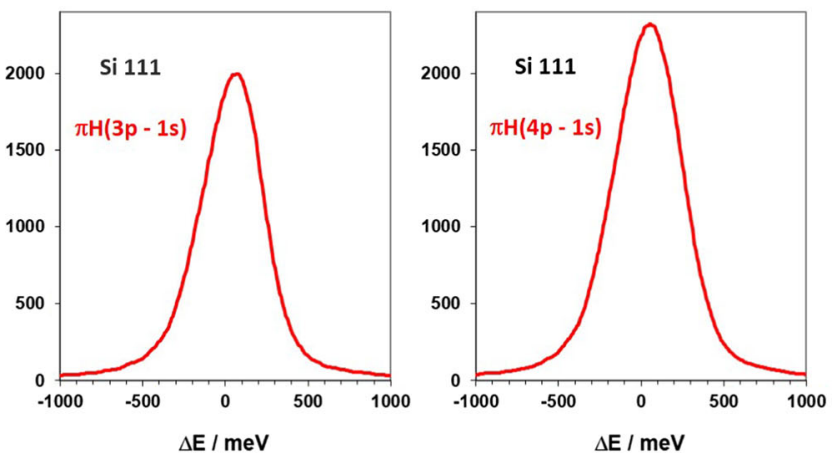

Entries are given for $1 / 10$ of the detector's pixel size or $4 \mu \mathrm{m}$ for $10^{6}$ Monte-Carlo events each
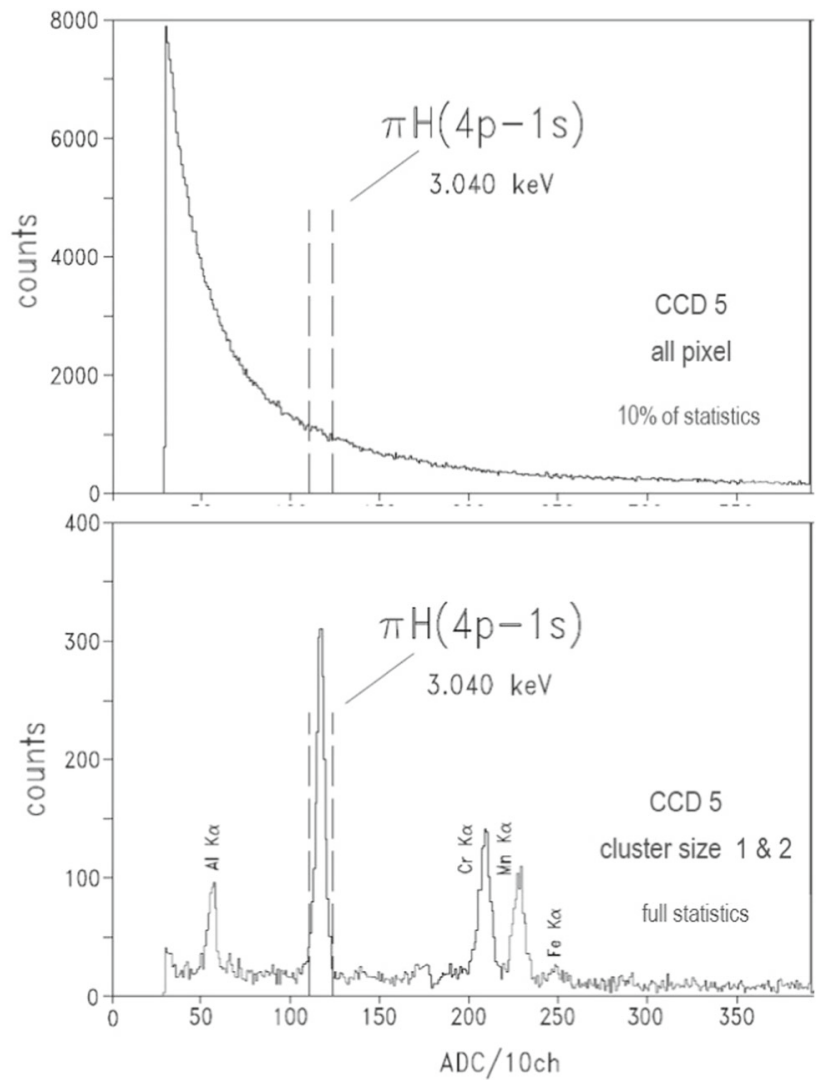

Fig. 8 Top: the charge spectrum of one CCD containing the Bragg reflection of the $\pi \mathrm{H}(4 p-1 s)$ transition. Bottom: the charge spectrum after rejecting all but 1- and 2-pixel events. Further cleaning is achieved by selecting a window around the charge range attributed to the $\pi \mathrm{H}$ line

uncertainty is negligibly small in view of the total line width of almost $1 \mathrm{eV}$. 


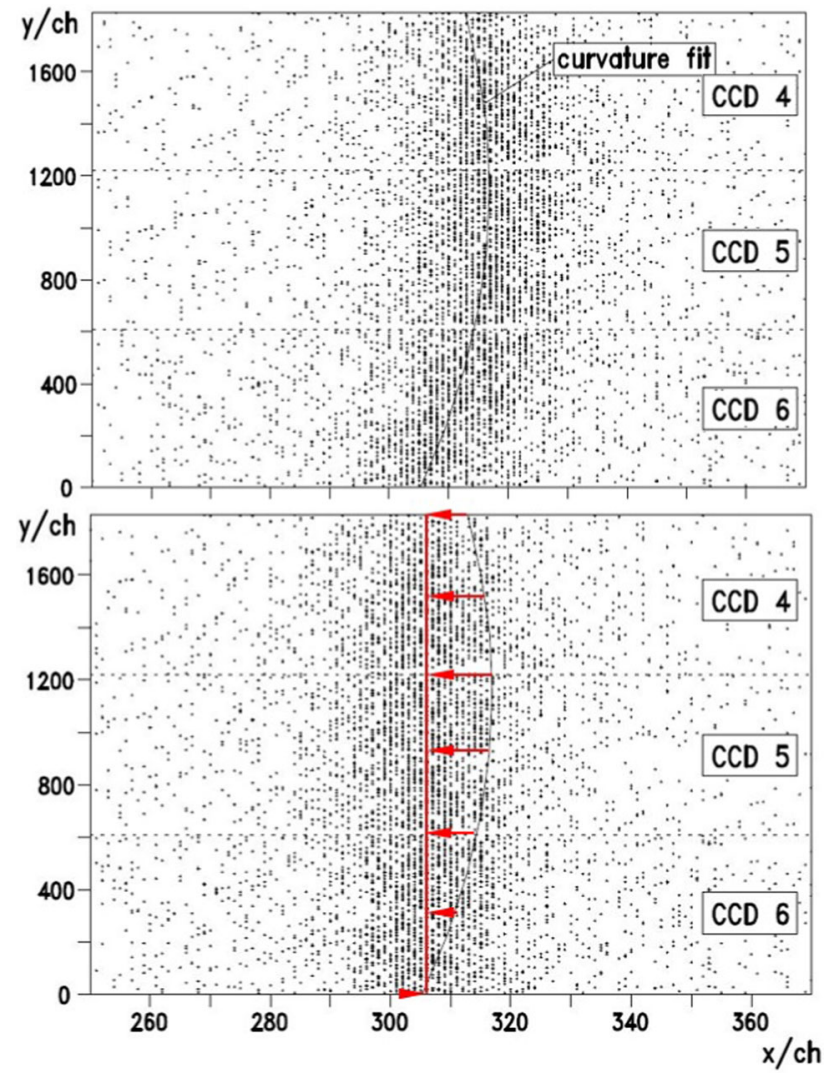

Fig. 9 Top: the hit pattern for the $\pi \mathrm{H}(4 p-1 s)$ transition in the detector plane after applying a pattern analysis. Bottom: the effect of the curvature correction by means of a parabolic fit. The window shown in the direction of dispersion $x$ comprises $1 / 12$ of the full detector range

\section{Data analysis}

The measured line profile $L P$ (see examples for the three transitions in Fig. 10) is formed by a convolution of the natural line width (given by a Lorentzian $L$ with width $\Gamma_{1 s}^{\pi \mathrm{H}}$ ), the spectrometer response $R$, and a sum of the Doppler profiles $D_{i}$ which correspond to the various kinetic-energy components originating from the Coulomb de-excitation. By exploiting distributivity, it can be written as
$L P=L \otimes R \otimes\left(\sum_{i} D_{i}\right)$

For the analysis of the spectra, both a frequentist and a Bayesian approach were used. Apart from trivial factors like intensity, position, and background level (assumed to be constant), the important parameters for the analysis are

- the hadronic width $\Gamma_{1 s}^{\pi \mathrm{H}}$ and

- the kinetic energy distribution given by the model chosen for $\sum_{i} D_{i}$.

\subsection{Modelling of the kinetic energy distribution}

The model used for the kinetic energy distribution is based on the results obtained from the muonic hydrogen experiment, where the line-shape model reduces to $L P_{\mu \mathrm{H}}=R_{\mu \mathrm{H}} \otimes\left(\sum_{i} D_{\mu \mathrm{H}, \mathrm{i}}\right)[32,33]$.

The distribution is built up by a number of narrow and discrete energy regions, $i$. $e$. energy intervals of a few eV width ("components" (Fig. 11)). The evaluation of muonic hydrogen showed that the quality of the description of the line shape does not depend on the width of the intervals representing the components of the kinetic-energy distribution, when the interval widths were varied between 1 and $8 \mathrm{eV}$.

Therefore, the low-energy component was fixed to an energy interval of $0-1$ or $0-2 \mathrm{eV}$ and for the width of the high-energetic components always a few $\mathrm{eV}$ was used. The central energies of the high-energy components and the relative intensities of all components were free parameters. The relative intensities were normalized to add up to $100 \%$. The construction of the corresponding Doppler spectra is described in detail in Ref. [137].

\subsection{Frequentist data analysis}

The spectra were fitted using the MINUIT package [142]. In a first Poisson likelihood $\chi^{2}$ optimization [143,144] it turned out that at least a low-energy component and in addition one
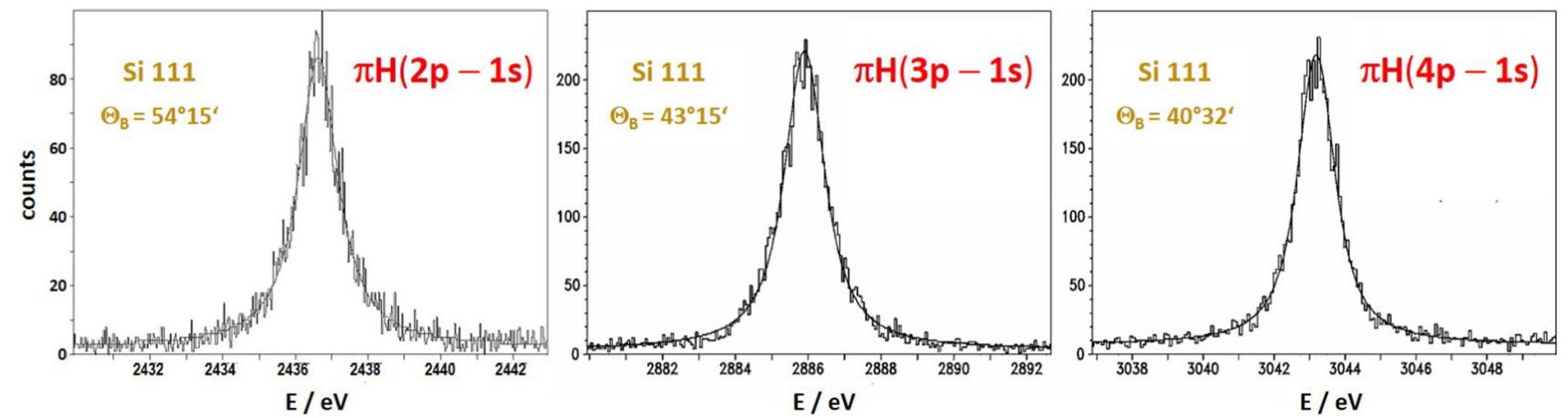

Fig. 10 The spectra of the $\pi \mathrm{H}(2-1)(\mathrm{A} 1), \pi \mathrm{H}(3-1)(\mathrm{B} 2)$, and $\pi \mathrm{H}(4-1)$ transitions (C1) measured at 10 bar equivalent target density 
Table 4 Relative intensity of the low- and high-energy components for the spectra with higher statistics as obtained in the 2-component fits in the frequentist approach

\begin{tabular}{|c|c|c|c|c|c|}
\hline \multirow[t]{2}{*}{ Label } & \multirow[t]{2}{*}{ Transition } & \multicolumn{2}{|c|}{ Low-energy } & \multicolumn{2}{|l|}{ High-energy } \\
\hline & & Interval & Rel. int. & Interval & Rel. int. \\
\hline $\mathrm{A} 1$ & $\pi \mathrm{H}(2 p-1 s)$ & {$[0-2]$} & $78 \pm 9$ & {$[154-162]$} & $22 \pm 9$ \\
\hline $\mathrm{A} 2$ & $\pi \mathrm{H}(2 p-1 s)$ & {$[0-1]$} & $84 \pm 5$ & {$[74-82]$} & $16 \pm 4$ \\
\hline B2 & $\pi \mathrm{H}(3 p-1 s)$ & {$[0-2]$} & $54 \pm 6$ & {$[68-72]$} & $46 \pm 6$ \\
\hline B3 & $\pi \mathrm{H}(3 p-1 s)$ & {$[0-2]$} & $71 \pm 10$ & {$[72-80]$} & $29 \pm 9$ \\
\hline $\mathrm{C} 1$ & $\pi \mathrm{H}(4 p-1 s)$ & {$[0-1]$} & $33 \pm 38$ & {$[12-16]$} & $67 \pm 38$ \\
\hline $\mathrm{C} 2$ & $\pi \mathrm{H}(4 p-1 s)$ & {$[0-1]$} & $59 \pm 11$ & {$[32-36]$} & $41 \pm 11$ \\
\hline
\end{tabular}

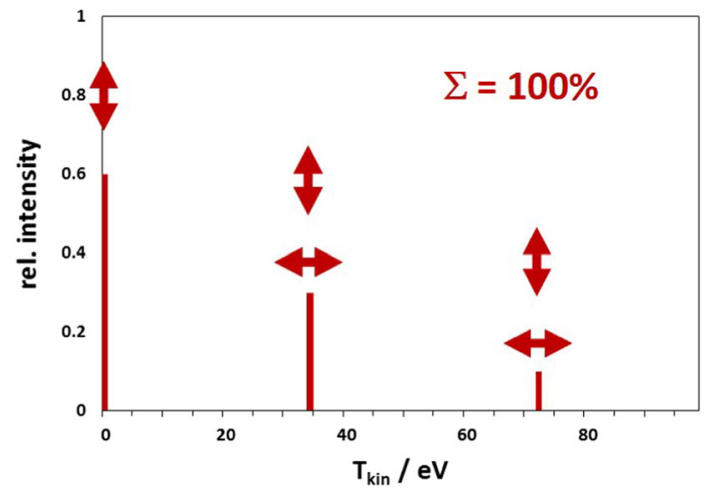

Fig. 11 Modelling of the kinetic energy distribution with three components. Free parameters are the relative intensities (normalized to 100\%) and the positions of the high-energy components

high-energy component are needed to achieve an acceptable $\chi^{2}$. For example, for the transition $\pi \mathrm{H}(2 p-1 s)$ with the highest statistics (A2 in Table 3), the fit with the low-energy component alone has $\chi^{2} /$ ndf $=1.2$, the fit with the highenergy component alone always yields $\chi^{2} /$ ndf $>3.0$, while the 2 -component fit has $\chi^{2} /$ ndf $=0.98$. The number of degrees of freedom is ndf $=554$.

The relative intensities of low- and high-energy component at minimal $\chi^{2}$ are shown in Table 4 for the most relevant transitions measured.

Such two-component analyses provided starting parameters for the 3-component fits including the second highenergy component. However, even in the case of the $(2-1)$ transition, a 3-component fit does not significantly improve the value of $\chi^{2}$. The weighted averages for $\Gamma_{1 s}^{\pi \mathrm{H}}$ as obtained from the 2- and 3-component fits are almost identical (Table 7).

The 2-component analyses of two low-statistics spectra at the extreme densities 3.9 bar and $\mathrm{LH}_{2}$ (measurements B1 and B4 (Table 3)) yielded $\Gamma_{1 s}^{\pi \mathrm{H}}=(890 \pm 150) \mathrm{meV}$ and $(815 \pm 109) \mathrm{meV}$ in good agreement with the result obtained for the other spectra. As the upper limit for the induced Stark width of $30 \mathrm{meV}$ at $\mathrm{LH}_{2}$ density [109] is much smaller than the statistical error, the corresponding correction is omitted for the measurement B4.
Table 5 The bias correction to be added to the frequentist fit result for $\Gamma_{1 s}^{\pi \mathrm{H}}$ as determined by Monte-Carlo simulations

\begin{tabular}{|c|c|c|c|}
\hline Label & Transition & bias/meV & \\
\hline belowruleskip & & 3-component fit & 2-component fit \\
\hline A1 & $\pi \mathrm{H}(2 p-1 s)$ & $-24 \pm 4$ & $-46 \pm 4$ \\
\hline A2 & $\pi \mathrm{H}(2 p-1 s)$ & $-20 \pm 2$ & $-13 \pm 1$ \\
\hline B2 & $\pi \mathrm{H}(3 p-1 s)$ & $-6 \pm 3$ & $6 \pm 2$ \\
\hline B3 & $\pi \mathrm{H}(3 p-1 s)$ & $-10 \pm 4$ & $6 \pm 2$ \\
\hline $\mathrm{C} 1$ & $\pi \mathrm{H}(4 p-1 s)$ & $-25 \pm 2$ & $-26 \pm 3$ \\
\hline $\mathrm{C} 2$ & $\pi \mathrm{H}(4 p-1 s)$ & $-34 \pm 2$ & $-37 \pm 3$ \\
\hline
\end{tabular}

For the kinetic energy models, the results corresponding to the minimal $\chi^{2}$ were used. The errors reflect statistical uncertainties only

As discussed in the earlier analysis of pionic hydrogen and deuterium data from this series of experiments [29,61, 145], the possible occurrence of a bias in the parameter determination must be considered [30], in particular, because the kinetic energy distribution can only roughly be approximated by a simple few-component model. Based on these results, a series of Monte-Carlo simulations has been performed for each case (400-800 per spectrum) in order to estimate the bias correction. The values are shown in Table 5.

\subsection{Bayesian data analysis}

The Bayesian analysis, though the evidence for a second high-energy component is weak, always used the 3component model for the $(2 p-1 s),(3 p-1 s)$, and $(4 p-1 s)$ transitions.

The evaluation procedure follows a suggestion by R. Trotta [146]. It uses a number of models inside the range of physically reasonable assumptions. Each of the models is subject to a Bayesian evaluation which leads to a value for the so-called Bayesian evidence, which is proportional to the probability of the model itself, as well as for a posterior distribution.

In the present case, 190 different 3-component models have been used for the $(2-1)$ transition. For the study of the $(3-1)$ and the $(4-1)$ transitions, a number of 78 and 36 models, respectively, turned out to be sufficient because 
Table 6 The schematics of kinetic energy distributions assumed for the $(2-1)$ transition with one low-energy (LE) and two high-energy components (HE) having a width of $1 \mathrm{eV}$ each

\begin{tabular}{|c|c|c|c|c|c|c|c|}
\hline \multirow{2}{*}{$\frac{\mathrm{LE} / \mathrm{eV}}{0-1}$} & \multirow{2}{*}{$\frac{1 . \mathrm{HE} / \mathrm{eV}}{10-11}$} & \multicolumn{6}{|c|}{ Corresponding set of $2 . \mathrm{HE} / \mathrm{eV}$} \\
\hline & & $20-21$ & $30-31$ & $40-41$ & $50-51$ & $\ldots$ & 200-201 \\
\hline $0-1$ & $20-21$ & & $30-31$ & $40-41$ & $50-51$ & $\ldots$ & 200-201 \\
\hline $0-1$ & $30-31$ & & & $40-41$ & $50-51$ & $\ldots$ & 200-201 \\
\hline . & . & & & & & . & \\
\hline . & . & & & & & . & \\
\hline . & . & & & & & . & \\
\hline $0-1$ & $180-181$ & & & & & $190-191$ & 200-201 \\
\hline
\end{tabular}

In total 190 parameter combinations ("models") are treated in a Bayesian evaluation of the smaller energy range. The distribution in energy of the three components was systematically varied over the physical range permitted, with the exception that the low-energy component was always fixed to the interval $0-1 \mathrm{eV}$. The scheme for the $(2-1)$ analysis is given in Table 6. As a test, for the $(2-1)$ transition the 190 models were extended from the upper energy of $200 \mathrm{eV}$ up to $300 \mathrm{eV}$. This resulted in a change in the value of $\Gamma_{1 s}^{\pi \mathrm{H}}$ of $2 \mathrm{meV}$ only.

Here, the advantage of this method comes into effect because for each of the models the value of the Bayesian evidence provides the corresponding weight of the model itself (proportional to its assigned probability) for averaging. In order to do this, the model with maximum evidence was determined and normalized to a value of 1 , leading then to lower values for the transformed evidences of all the other models. These lower values are the measure by which the number of points in the posterior distribution for the parameter (here $\Gamma_{1 s}^{\pi \mathrm{H}}$ ) is reduced for each model. This was done by a Monte Carlo routine. In a final step, the different weighted posterior distributions were summed up. From this final distribution, the values for the mean of $\Gamma_{1 s}^{\pi \mathrm{H}}$ and its uncertainty as given in Table 7 were found.

\subsection{Comparison of frequentist and Bayesian results}

The results for the width $\Gamma_{1 s}^{\pi \mathrm{H}}$ from the individual spectra are compared in Table 7. The weighted average values are in good agreement with each other where the associated uncertainty can be regarded as the statistical error of the data available. An estimate for the systematic contribution to the error of the final average value is derived in Sect. 5.5.3.

Though we can conclude from the results given in Table 7 that the assumptions about the bias correction are reasonable, the frequentist fit can suffer from the particular assumptions for the kinetic energy distribution. In view of this uncertainty, the Bayesian analysis with its conservative error estimate is chosen for the final result.
Table 7 Comparison of results for the width $\Gamma_{1 \mathrm{~s}}^{\pi \mathrm{H}}$ (in meV) for the 3component Bayesian and the 2- and 3-component frequentist approach for the various experimental conditions (Table 3)

\begin{tabular}{lllll}
\hline Label & Transition & Bayesian & \multicolumn{2}{l}{ Frequentist fit } \\
\cline { 4 - 5 } & & 3-comp. & 3-comp. & 2-comp. \\
\hline A1 & $\pi \mathrm{H}(2 p-1 s)$ & $864 \pm 61$ & $876 \pm 92$ & $838 \pm 78$ \\
A2 & $\pi \mathrm{H}(2 p-1 s)$ & $895 \pm 21$ & $875 \pm 24$ & $889 \pm 25$ \\
B1 & $\pi \mathrm{H}(3 p-1 s)$ & - & - & $890 \pm 150$ \\
B2 & $\pi \mathrm{H}(3 p-1 s)$ & $746 \pm 48$ & $753 \pm 50$ & $746 \pm 43$ \\
B3 & $\pi \mathrm{H}(3 p-1 s)$ & $889 \pm 44$ & $894 \pm 50$ & $877 \pm 51$ \\
B4 & $\pi \mathrm{H}(3 p-1 s)$ & - & - & $815 \pm 109$ \\
$\mathrm{C} 1$ & $\pi \mathrm{H}(4 p-1 s)$ & $793 \pm 57$ & $829 \pm 72$ & $785 \pm 79$ \\
C2 & $\pi \mathrm{H}(4 p-1 s)$ & $753 \pm 51$ & $758 \pm 75$ & $745 \pm 79$ \\
Average statistical & $856 \pm 16$ & $852 \pm 18$ & $846 \pm 18$ \\
Systematic error & \pm 22 & \pm 13 & \pm 14 \\
Experiment total & $\mathbf{8 5 6} \pm \mathbf{2 7}$ & $852 \pm 22$ & $846 \pm 23$
\end{tabular}

5.5 Systematic uncertainties

\subsubsection{Influence on $\Gamma_{1 \mathrm{~s}}^{\pi \mathrm{H}}$ of cuts applied to the data}

In order to estimate systematic effects in the evaluation of the data, the influence of the following parameters has been studied:

1. Window applied to the CCD charge spectrum (see Fig. 8): A variation of the window between $2 / 3$ and $4 / 3$ of FWHM changes the value of $\Gamma_{1 s}^{\pi \mathrm{H}}$ by less than $50 \%$ of its statistical error for the corresponding spectrum. The average variation of $\Gamma_{1 s}^{\pi \mathrm{H}}$ was found to be $\left(\begin{array}{c}+3 \% \\ -2 \%\end{array}\right)$ and includes both the scattering of the result for all spectra and their statistical errors. The insensitivity is in line with the observation that the background levels obtained in the analyses scale perfectly with the width of the charge window.

2. Fit interval: The maximal interval width is given by the size of one CCD device and amounts to 44,36 , and $18 \mathrm{eV}$ for the $(4-1),(3-1)$, and $(2-1)$ transition, respectively. 
The value of $\Gamma_{1 s}^{\pi \mathrm{H}}$ stabilizes when the interval containing the peak is $15 \mathrm{eV}$ or larger. Smaller intervals resulted in too large values for $\Gamma_{1 s}^{\pi \mathrm{H}}$ because the interval boundaries then cut into the tails of the line. This could be proven by an evaluation of the $(3-1)$ and $(4-1)$ transitions by systematically shortening the fit intervals to values smaller than 36 or $44 \mathrm{eV}$, respectively.

3. Fluctuation of data subsets: The total measuring time for an individual transition spanned from 3 to 25 days. Therefore, subsets of the larger periods were evaluated separately. No time dependence of the result for $\Gamma_{1 s}^{\pi \mathrm{H}}$ could be found.

\subsubsection{Influence of the kinetic energy distribution}

It is tempting to use the theoretical prediction for the kinetic energy distributions, like the ones shown in Fig.3, for $\left(\sum_{i} D_{i}\right)$ in Eq. (19). The corresponding results for $\Gamma_{1 s}^{\pi \mathrm{H}}$ are in the range $781-963 \mathrm{meV}$. The statistical errors are only $\pm 7 \mathrm{meV}$ for the measurement with the highest statistics (measurement A2) and $\pm(17-20)$ meV else (A1, B2, B3, C1, C2). Such small uncertainties are due to the fact that this analysis assumes no error for the kinetic energy distributions. Therefore, we emphasize that these values for $\Gamma_{1 s}^{\pi \mathrm{H}}$ and their errors do not constitute experimental result.

The above-mentioned range of the results for $\Gamma_{1 s}^{\pi \mathrm{H}}$, which is much larger than the individual errors, yields an uncertainty of about $40 \mathrm{meV}$ for the average of the six measurements. We interpret this as an incomplete matching of theoretical description and data.

To quantify this mismatch, results from the muonichydrogen analysis are used, where it was possible to reconstruct the coarse structure of the kinetic energy distribution by de-convolution. This revealed that experiment and cascade theory agree within about $3 \sigma$ and, in particular, that the intensity of the low-energy component is reproduced with a value of about $20 \%$ lower than theoretically predicted. Such a de-convolution turned out to be not feasible for pionic hydrogen because the additional parameter Lorentzian broadening, $\Gamma_{1 s}^{\pi \mathrm{H}}$ (Eq. (19)), hides any detail.

To estimate the order of magnitude of the mismatch on $\Gamma_{1 s}^{\pi \mathrm{H}}$, the strength of the low-energetic component was reduced for the model distributions to values of $80 \%$ compared to the values of the theoretical distributions as shown in Fig. 3. For this study, the six spectra (2 for each of the transitions $(n p-1 s))$ with the highest accumulated statistics were used (measurements A1, A2, B2, B3, C1, C2).

In the case of the $(2 p-1 s),(3 p-1 s),(4 p-1 s)$ transition, the change of the strength of the low-energy component reduces the value for $\Gamma_{1 s}^{\pi \mathrm{H}}$ by 20,26 , and $12 \mathrm{meV}$, respectively. The differences are consistent with the systematic error derived from the scattering of the results from the cascade-model-free analyses (Table 7).

\subsubsection{Estimate of systematic error}

From the discussion in Sect.5.5.2 it can be concluded that details of the kinetic energy distribution, which are not accessible to our analysis, are important for the scattering of the results for $\Gamma_{1 s}^{\pi \mathrm{H}}$ (Table 7). The method recommended by the Particle-data-Group is to quantify such a scattering by the $\chi^{2}$-like quantity $S=\chi^{2} /(n-1)$, where $\chi^{2}=\sum_{i}^{n} \frac{\left(x_{i}-\bar{x}\right)^{2}}{\sigma_{i}^{2}}$, $x_{i}$ and $\sigma_{i}$ are the individual results and their uncertainties, and $\bar{x}$ the weighted average of the $n$ measurements [2]. The total uncertainty is then given by the average statistical error multiplied by the enhancement factor $\sqrt{S}$.

One can get an estimate for the systematic error by subtracting quadratically the average statistical error from the total error (Table 7 - systematic error). It turns out, that the range of values obtained in this way is close to the scattering found from the variation of the fraction of the low-energy component of the kinetic energy distribution.

\section{Results}

The final result for $\Gamma_{1 s}^{\pi \mathrm{H}}$ of the experiment series described here, taken from the Bayesian analysis, is

$\Gamma_{1 s}^{\pi \mathrm{H}}=856 \pm 16_{\text {stat }} \pm 22_{\text {sys }} \mathrm{meV}$.

The total experimental error obtained by quadratic addition amounts to $\triangle \Gamma_{1 s, \exp }^{\pi \mathrm{H}}= \pm 27 \mathrm{meV}$ or $\pm 3.1 \%$.

\subsection{Scattering lengths}

Using Eq. (3) one gets the scattering length for the charge exchange reaction:

$a_{\pi^{-} p}^{\mathrm{cex}}=(-124.4 \pm 2.0) \cdot 10^{-3} m_{\pi}^{-1}( \pm 1.6 \%)$.

Applying the correction $\triangle a_{\pi^{-} p}^{\text {cex }}$ from [38,51] (Eq. (10)) in Eq. (8) one obtains the isovector scattering length:

$a^{-}=\left(88.2 \pm 1.4_{\mathrm{exp}} \pm 0.6_{\mathrm{th}}\right) \cdot 10^{-3} m_{\pi}^{-1}( \pm 1.7 \%)$.

The second error reflects the accuracy in the theoretical calculation of $\triangle a_{\pi^{-} p}^{\text {cex }}$.

The $\pi^{-} p$ scattering length $a_{\pi^{-}}$has been determined from the hadronic shift in pionic hydrogen (Eqs. (1) and (4)) in $[27,28]$ :

$a_{\pi^{-} p}=(85.26 \pm 0.12) \cdot 10^{-3} m_{\pi}^{-1}$.

After applying the correction $\triangle a_{\pi^{-}}$(Eqs. (9) and (11)) one gets

$a^{+}+a^{-}=\left(93.4 \pm 0.12_{\exp } \pm 2.8_{\mathrm{th}}\right) \cdot 10^{-3} m_{\pi}^{-1}$.

This improved value in comparison with [28] is due to a newer result for the LEC $c_{1}$ [21]. 


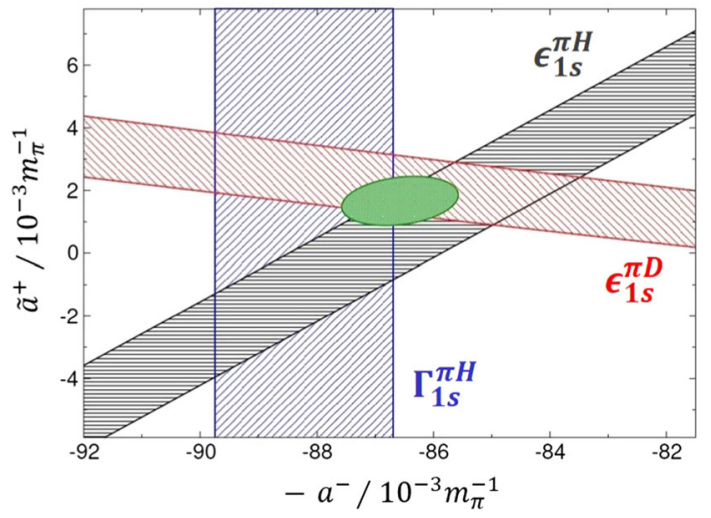

Fig. 12 Constraints on the scattering lengths $\tilde{a}^{+}$and $a^{-}$by the hadronic $1 s$ level shift $\epsilon_{1 s}^{\pi \mathrm{H}}$ in pionic hydrogen, $\epsilon_{1 s}^{\pi \mathrm{D}}$ in pionic deuterium, and the $1 s$ level broadening $\Gamma_{1 s}^{\pi \mathrm{H}}$ in pionic hydrogen as obtained within the framework of $[21,51,57,147]$. The ellipse displays the combined constraints as given in Table 8 ( $3^{r d}$ line)

As already mentioned, three experimental results, $\epsilon_{1 s}^{\pi \mathrm{H}}$, $\Gamma_{1 s}^{\pi \mathrm{H}}$, and $\epsilon_{1 s}^{\pi \mathrm{D}}$, are available to determine the two quantities $a^{+}$and $a^{-}$. Table 8 compares the results obtained from different data sets: only the pionic hydrogen $\left(1^{s t}\right.$ line $)$, only the shift data with $a^{-}$dependent corrections ( $2^{\text {nd }}$ line), and after applying a constraint calculation with all three results $\left(3^{r d}\right.$ line).

The errors of $a^{+}$and with it $a^{+}+a^{-}$(Eqs. (22) and (24)) are dominated by the uncertainty of the chiral corrections, which is mainly due to the poor knowledge of the LEC $f_{1}$ that appears in combination with the LEC $c_{1}$ and $a^{+}$ itself. Therefore, in theoretical analyses the corresponding combination denoted $\tilde{a}^{+}$is more convenient, which has a much smaller uncertainty $[51,57]$ (Table 8 ). The most recent $\chi$ PT calculation provides for the difference $\tilde{a}^{+}-a^{+}=$ $(-6.1 \pm 2.5) \cdot 10^{-3} m_{\pi}^{-1}$ [21]. In contrast, the uncertainty of $a^{-}$is dominated by the experimental error.

Given the final values of the three experiments, in view of the larger uncertainty of $\Gamma_{1 s}^{\pi \mathrm{H}}$, the values of $a^{+}$and $a^{-}$are essentially determined by the results derived from the energy shifts $\epsilon_{1 s}^{\pi \mathrm{H}}$ and $\epsilon_{1 s}^{\pi \mathrm{D}}$. Therefore, discarding the constraint from $\Gamma_{1 s}^{\pi \mathrm{H}}$ leads to marginal changes only. It is worthwhile to note that $a^{+}$remains positive by 3 standard deviations.

Figure 12 shows the constraints imposed on the scattering lengths $\tilde{a}^{+}$and $a^{-}$by the three pionic-atom results. It constitutes the update of the analyses of $[21,51,57,147]$ for the new result for $\Gamma_{1 s}^{\pi \mathrm{H}}$.

The $\pi N$ scattering lengths directly accessible from pionic-atom experiments via $\Gamma_{1 s}^{\pi \mathrm{H}}$ and $\epsilon_{1 s}^{\pi \mathrm{H}}$ are $a_{\pi^{-} p}^{\text {cex }}$ and $a_{\pi^{-}}$(Eqs. (3) and (4)). In order to quantify the isospinbreaking corrections via the triangle relation (Eq. (15)), the scattering-length difference $a_{\pi^{-}} p-a_{\pi^{+} p}$ is needed. In an approach chosen by $[51,63]$, emphasising the experimental information for $\epsilon_{1 s}^{\pi \mathrm{H}}$ together with corrections depending amongst others on $\epsilon_{1 s}^{\pi \mathrm{D}}$ and $a^{-}$as derived from $\Gamma_{1 s}^{\pi \mathrm{H}}$, the scattering-length difference can be written as

$a_{\pi^{-} p}-a_{\pi^{+} p}=2 \cdot a_{\pi^{-} p}+\triangle a\left(\epsilon_{1 s}^{\pi \mathrm{D}}, a^{-}\right)$.

Numerically, the scattering length difference is given to $97 \%$ by the experimental results for $a_{\pi^{-}}$, i.e. the result is very robust against the input for $\triangle a\left(\epsilon_{1 s}^{\pi \mathrm{D}}, a^{-}\right)$. The correction term worked out in the framework of the chiral expansion has the form $[38,51,57]$

$$
\begin{aligned}
\triangle a\left(\epsilon_{1 s}^{\pi \mathrm{D}}, a^{-}\right) & =-2 \cdot\left(\tilde{a}^{+}+\Delta \tilde{a}^{+}\right)+\left(\Delta a_{\pi^{-} n}-\Delta a_{\pi^{+}}\right) \\
& =(2.1 \pm 1.7) \cdot 10^{-3} m_{\pi}^{-1} .
\end{aligned}
$$

This correction involves the new result for $\Gamma_{1 s}^{\pi \mathrm{H}}$.

Using the scattering lengths Eqs. (21) and (23) together with the correction given in Eq. (26) yields for the triangle relation

$R=(-1.9 \pm 1.9) \%$.

The result might be seen in slight tension with the $\chi \mathrm{PT}$ calculation, $R=(+1.5 \pm 1.1) \%$ [38], but it overlaps well with the value obtained from the analysis of low-energy $\pi N$ scattering data yielding $R=(-2.6 \pm 4.4) \%$ [85].

Finally, a recent extraction of the $\pi N$ scattering lengths from the low-energy $\pi N$ scattering found $a_{\pi^{-}}=(83.3 \pm$ 2.1) $\cdot 10^{-3} m_{\pi}^{-1}, a_{\pi^{+}}=(-85.7 \pm 4.2) \cdot 10^{-3} m_{\pi}^{-1}$, and $a_{\pi^{-} p \rightarrow \pi^{0} n}=(-122.6 \pm 4.1) \cdot 10^{-3} m_{\pi}^{-1}$ [85]. Using Eq. (25) for the pionic-atom results, one gets

$a_{\pi^{+} p}=(-87.4 \pm 1.7) \cdot 10^{-3} m_{\pi}^{-1}$.

In other words, recent $\pi H, \pi D$, and low-energy $\pi N$ scattering data are quantitatively highly consistent when analysed within the framework of $\chi$ PT.

\subsection{Related quantities}

In order to determine the $\pi N$ coupling via the GMO sum rule (Eq. (16)), the scattering-length difference $a_{\pi^{-}}-a_{\pi^{+}} p$ must be corrected for virtual-photon contributions $[51,85]$. Starting from Eq. (25) one gets

$$
\begin{aligned}
a_{\pi^{-} p}^{\gamma}-a_{\pi^{+} p}^{\gamma} & =a_{\pi^{-} p}-a_{\pi^{+} p}-(2.1 \pm 1.8) \cdot 10^{-3} m_{\pi}^{-1} \\
& =(170.7 \pm 2.5) \cdot 10^{-3} m_{\pi}^{-1} .
\end{aligned}
$$

Alternatively, the scattering length difference can be expressed as

$a_{\pi^{-} p}-a_{\pi^{+} p}=-\sqrt{2} \cdot a_{\pi^{-} p}^{\mathrm{cex}}+\triangle a\left(\Gamma_{1 s}^{\pi \mathrm{H}}\right)$.

Here, the experimental result for $\Gamma_{1 s}^{\pi \mathrm{H}}$ dominates the numerical value by $99 \%$. With the appropriate correction to include also virtual-photon effects, which depends among others on 
Table 8 Isoscalar and isovector $\pi N$ scattering lengths $a^{+}$and $a^{-}$as derived from various combinations of $\pi \mathrm{H}$ and $\pi \mathrm{D}$ data. $\tilde{a}^{+}$is a combination of the LECs $f_{1}$ and $c_{1}$ with $a^{+}[51,54,57]$

Table 9 Results for pion-nucleon coupling constant $\frac{g_{c}^{2}}{4 \pi}$, the Goldberger-Treiman discrepancy $\triangle G T$, and the threshold amplitude for $\pi^{-}$ photoproduction $E_{0+}^{\mathrm{thr}}\left(\pi^{-} p\right)$

\begin{tabular}{llcc}
\hline Source & $a^{+} / 10^{-3} m_{\pi}^{-1}$ & $a^{-} / 10^{-3} m_{\pi}^{-1}$ & $\tilde{a}^{+} / 10^{-3} m_{\pi}^{-1}$ \\
\hline$\epsilon_{1 s}^{\pi \mathrm{H}}+\Gamma_{1 s}^{\pi \mathrm{H}}$ & $5.2 \pm 3.1$ & $88.2 \pm 1.7$ & $-0.9 \pm 1.8$ \\
$\epsilon_{1 s}^{\pi \mathrm{H}}+\epsilon_{1 s}^{\pi \mathrm{D}}$ & $8.0 \pm 2.6$ & $85.3 \pm 1.3$ & $1.9 \pm 0.8$ \\
$\epsilon_{1 s}^{\pi \mathrm{H}}+\Gamma_{1 s}^{\pi \mathrm{H}}+\epsilon_{1 s}^{\pi \mathrm{D}}$ & $7.8 \pm 2.6$ & $86.6 \pm 1.0$ & $1.7 \pm 0.8$ \\
\hline
\end{tabular}

Using the constraint given by $\epsilon_{1 s}^{\pi \mathrm{D}}$, the full information available from pionic hydrogen and deuterium is exploited and, therefore, constitutes the final result of this experiment series ( $3^{\text {rd }}$ line)

\begin{tabular}{|c|c|c|c|c|}
\hline & & $\frac{g_{c}^{2}}{4 \pi}$ & $\triangle G T / \%$ & $E_{0+}^{\mathrm{thr}}\left(\pi^{-} p\right) / 10^{-3} m_{\pi}^{-1}$ \\
\hline$\epsilon_{1 s}^{\pi \mathrm{H}}+\epsilon_{1 s}^{\pi \mathrm{D}}$ Eq. (29) & & $13.66 \pm 0.20^{a}$ & $0.9 \pm 0.7^{b}$ & $-32.0 \pm 0.6$ \\
\hline$\Gamma_{1 s}^{\pi \mathrm{H}}$ Eq. $(31)$ & & $13.96 \pm 0.22^{a}$ & $2.0 \pm 0.8^{b}$ & $-32.3 \pm 0.7$ \\
\hline$\pi N$ DR $B^{+}$ & [148] & $13.74 \pm 0.10$ & & \\
\hline$\pi N \mathrm{DR}$ & [149] & $13.76 \pm 0.01^{c}$ & & \\
\hline$N N$ scatt. & [150] & $13.54 \pm 0.05^{c}$ & $1.4 \pm 0.9$ & \\
\hline QCD sum rules & [151] & & $1.5-2.2$ & \\
\hline$N N$ scatt. & [152] & $13.97 \pm 0.11$ & & \\
\hline$N N$ EFT & [153] & $13.92 \pm 0.09$ & & \\
\hline $\exp . \pi^{-} p \rightarrow \gamma n$ & [154] & & & $-31.5 \pm 0.8$ \\
\hline fixed- $t$ DR & [155] & & & $-31.7 \pm 0.2$ \\
\hline
\end{tabular}

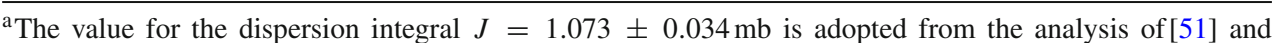
contributes (by quadratic addition) with about $50 \%$ to the total error

${ }^{\mathrm{b}}$ Using $f_{\pi}=(92.28 \pm 0.09) \mathrm{MeV}[2]$ and $g_{A}=1.27641 \pm 0.00056[156]$

${ }^{\mathrm{c}}$ Errors reflect mainly statistical uncertainties

The two sets of values derived from pionic atoms, combining $\epsilon_{1 s}^{\pi \mathrm{H}}$ with $\epsilon_{1 s}^{\pi \mathrm{D}}$ and from $\Gamma_{1 s}^{\pi \mathrm{H}}$, are compared to the ones obtained from pion-nucleon $(\pi N)$ dispersion-relation (DR) applications and more recent analyses of nucleon-nucleon $(N N)$ scattering data. The results for $E_{0+}^{\text {thr }}\left(\pi^{-} p\right)$ include the chiral correction $(-1.0 \pm$ $0.6) \cdot 10^{-3} m_{\pi}^{-1}$ as given by [71] the chiral prediction for $R$ [147], the result is

$$
\begin{aligned}
a_{\pi^{-} p}^{\gamma}-a_{\pi^{+} p}^{\gamma} & =-\sqrt{2} \cdot a_{\pi^{-} p}^{\text {cex }}+(0.7 \pm 1.0) \cdot 10^{-3} m_{\pi}^{-1} \\
& =(176.6 \pm 3.0) \cdot 10^{-3} m_{\pi}^{-1} .
\end{aligned}
$$

Equations (29) and (31) represent the same physical quantity derived from the two practically independent pionic-atom results: on the one hand from $\epsilon_{1 s}^{\pi \mathrm{H}}$ together with $\epsilon_{1 s}^{\pi \mathrm{D}}$ and on the other from $\Gamma_{1 s}^{\pi \mathrm{H}}$. They agree with each other within $2 \sigma$. This comparison constitutes an important check of consistency for the pionic-atom data.

Table 9 shows the corresponding two sets of values for the pion-nucleon coupling constant $\frac{g_{c}^{2}}{4 \pi}$ via the GMO sum rule, the Goldberger-Treiman discrepancy $\triangle G T$ (Eq.(17)), and the threshold electric dipole amplitude $E_{0+}^{\mathrm{thr}}\left(\pi^{-} p\right)$ (Eq. (18)). Within the experimental and theoretical uncertainties, the slight tension between the two sets of results does not constitute any contradiction. The results show also good agreement with more recent values obtained from the analyses of $\pi N, N N$, and photoproduction data.

\section{$6.3 \pi \mathbf{N} \sigma$-term}

Within the constraints imposed by an analysis using the RoySteiner equations, the $\pi N \sigma$-term reads in terms of the $\pi N$ scattering lengths in the isospin basis $I=1 / 2$ and $3 / 2$ [83]

$$
\begin{aligned}
\sigma_{\pi N} & =(59.1 \pm 3.1) \mathrm{MeV}+\sum_{I} c_{I} \cdot\left(a_{I}-\bar{a}_{I}\right) \\
c_{1 / 2} & =0.24 \mathrm{MeV} \cdot 10^{3} m_{\pi}, c_{3 / 2}=0.89 \mathrm{MeV} \cdot 10^{3} m_{\pi} .
\end{aligned}
$$

The reference values $\bar{a}_{I}$ for the scattering lengths, corrected for virtual photon effects, were extracted from the pionicatom results for $\epsilon_{1 s}^{\pi \mathrm{H}}, \Gamma_{1 s}^{\pi \mathrm{H}}$, and $\epsilon_{1 s}^{\pi \mathrm{D}}$ to be $\bar{a}_{1 / 2}=(169.8 \pm$ $2.0) \cdot 10^{-3} m_{\pi}^{-1}$ and $\bar{a}_{3 / 2}=(-86.3 \pm 1.8) \cdot 10^{-3} m_{\pi}^{-1}$ [85]. Here, the preliminary result for $\Gamma_{1 s}^{\pi \mathrm{H}}$ of $(823 \pm 19) \mathrm{meV}$ was used [145].

Adjusting the scattering lengths to the new value for $\Gamma_{1 s}^{\pi \mathrm{H}}$ yields $a_{1 / 2}=(169.9 \pm 2.0) \cdot 10^{-3} m_{\pi}^{-1}$ and $a_{3 / 2}=(-86.5 \pm$ 1.8) $\cdot 10^{-3} m_{\pi}^{-1}$, which constitutes a marginal change only. Consequently, the new pionic-atom based result,

$\sigma_{\pi N}=(59.0 \pm 3.5) \mathrm{MeV}$, 
is fully consistent with $\sigma_{\pi N}=(58 \pm 5) \mathrm{MeV}$ obtained from the analysis of low-energy $\pi N$ scattering data $[21,84,85]$. The discrepancy to the result $\sigma \approx 45 \mathrm{MeV}$ of the pioneering Karlsruhe-Helsinki phase-shift analysis [157,158] can be traced back to an unrealistically large value $a_{3 / 2}=$ $(-101 \pm 4) \cdot 10^{-3} m_{\pi}^{-1}$ related to the data base available at that time [85]. However, the discrepancy of about $20 \mathrm{MeV}$ between the experiment based values and lattice calculations remains [88-91].

\section{Summary}

The strong-interaction width $\Gamma_{1 s}^{\pi \mathrm{H}}$ of the ground state of pionic hydrogen has been determined from the line shape of the $\mathrm{X}$-ray transitions $(2 p-1 s),(3 p-1 s)$, and $(4 p-1 s)$ for $\mathrm{H}_{2}$ densities ranging from 4 bar (effective) to liquid. Using different transitions, a consistency check can be done on the influence of the density-dependent Doppler broadening due to Coulomb de-excitation, which is the main obstacle for a high-precision determination of $\Gamma_{1 s}^{\pi \mathrm{H}}$.

Compared to the previous experiment [24,25], which measured the $(3 p-1 s)$ transition at one target density only, the uncertainty for $\Gamma_{1 s}^{\pi \mathrm{H}}$ was reduced to about $50 \%$ of the one quoted there. The scattering of our results, however, demonstrates that the measurement of one transition at one density cannot avoid the noticeable errors arising from the complexity of the corrections for the Doppler broadening, even though the background levels have been reduced by factors up to 50 in the present series of measurements.

Both the frequentist approach as well as the Bayesian analysis clearly show the presence of a distinctive lowenergy component of the kinetic energy distribution containing about $2 / 3$ of the total intensity. The complex structure of the kinetic energy spectrum at higher energies can be effectively modelled by one or two high-energy components for the present experimental statistics. The details of these components are not critical for the numerical result, however, the accuracy for $\Gamma_{1 s}^{\pi \mathrm{H}}$ is limited to about $3 \%$. To summarize the current situation one can assert that the theoretical prediction for the kinetic energy distributions and the experimental results do not show any drastic inconsistencies.

Taking into account the difficulties related to the lineshape corrections due to Coulomb-de-excitation, future experiments should preferably use X-ray transitions from higher $n p$ initial states where the Doppler broadening is expected to be smaller. Since the accelerator currents improve continuously with time, future measurements with much improved statistics for the $(4-1)$ or even $(5-1)$ transition will become feasible. In this respect it is important to emphasize that for measurements using Johann-type setups, the coverage of a sufficiently large energy interval, i.e. a sufficiently large detector being equivalent to choose a well adapted dispersion, is essential to fix the background on both sides of the line. In this way, an accuracy of $1 \%$ or better can be achieved for the hadronic width of pionic hydrogen.

Acknowledgements We thank C. Hanhart and M. Hoferichter for discussions on various issues related to the scattering lengths and the update of the constraint calculation. We are grateful to N.Dolfus, H.Labus, B. Leoni, L. Stohwasser, and K.-P. Wieder for solving numerous technical problems as well as N. Nelms for the help in X-ray detector issues. Efforts are acknowledged of the PSI staff to provide excellent beam conditions and of the Carl Zeiss AG, Oberkochen, Germany, in the fabrication of the Bragg crystals. We thank Prof. Dr. E. Förster and his collaborators at the University of Jena and A. Freund and his group at ESRF for the help in characterising the crystal material as well as R. Lötzsch and I. Uschmann for a check of rocking curve calculations. We are indebted to PSI for supporting the stay during the run periods (D.F. A. and Y.-W.L.). Partial funding was granted by the Allianz Program of the Helmholtz Association contract no. EMMI HA-216 "Extremes of Density and Temperature: Cosmic Matter in the Laboratory" (P.I. and M. T.). A substantial part of this work is covered by the $\mathrm{PhD}$ thesis of one of us (A.H., Technische Universität Wien, 2008).

Funding Open Access funding enabled and organized by Projekt DEAL.

Data Availability Statement This manuscript has no associated data or the data will not be deposited. [Authors' comment: The datasets generated during and/or analysed during the current study are available from the corresponding author on reasonable request.]

Open Access This article is licensed under a Creative Commons Attribution 4.0 International License, which permits use, sharing, adaptation, distribution and reproduction in any medium or format, as long as you give appropriate credit to the original author(s) and the source, provide a link to the Creative Commons licence, and indicate if changes were made. The images or other third party material in this article are included in the article's Creative Commons licence, unless indicated otherwise in a credit line to the material. If material is not included in the article's Creative Commons licence and your intended use is not permitted by statutory regulation or exceeds the permitted use, you will need to obtain permission directly from the copyright holder. To view a copy of this licence, visit http://creativecomm ons.org/licenses/by/4.0/.

\section{References}

1. S. Weinberg, Physica A 96, 327 (1979)

2. M. Tanabashi et al., PDG. Phys. Rev. D 98, 030001 (2018)

3. M. Gell-Mann, R. Oakes, B. Renner, Phys. Rev. 122, 2195 (1968)

4. J. Gasser, H. Leutwyler, Ann. Phys. 158, 142 (1984)

5. J. Gasser, H. Leutwyler, Nucl. Phys. B 250, 465 (1985)

6. V. Bernard, N. Kaiser, Ulf-G. Meissner, Int. J. Mod. Phys. E 4, 193 (1995), and references therein

7. G. Ecker, Prog. Part. Nucl. Phys. 35, 1 (1995). and references therein

8. J. Gasser et al., Phys. Rev. D 64, 016008 (2001)

9. G. Colangelo, J. Gasser, H. Leutwyler, Nucl. Phys. B 603, 125 (2001)

10. B. Adeva et al., Phys. Lett B 619, 50 (2005)

11. CERN experiment PS212, https://home.cern/science/ experiments/dirac

12. A.W. Thomas, W. Weise, The Structure of the Nucleon (WILEYVCH, Berlin, 2001) 
13. S. Scherer, 'Introduction to Chiral Perturbation Theory', Advances in Nuclear Physics 27, ed. by J.W. Negele, E.W. Vogt (Springer, 2003)

14. E. Jenkins, A.V. Manohar, Phys. Lett. B 255, 558 (1991)

15. V. Bernard et al., Nucl. Phys. B 388, 315 (1992)

16. V. Bernard et al., Phys. Rev. C 52, 2185 (1995)

17. N. Fettes, U.-G. Meißner, Nucl. Phys. A 676, 311 (2000)

18. N. Fettes, U.-G. Meißner, Nucl. Phys. A 693, 693 (2001)

19. D. Gotta, Prog. Part. Nucl. Phys. 52, 133 (2004)

20. J. Gasser, V.E. Lyubovitzkij, A. Rusetski, Phys. Rep. 456, 167 (2008). and references therein

21. M. Hoferichter et al., Phys. Rep. 625, 1 (2016). and references therein

22. D. Sigg et al., Phys. Rev. Lett. 75, 3245 (1995)

23. D. Sigg et al., Nucl. Phys. A 609, 269 (1996)

24. H-Ch. Schröder et al., Phys. Lett. B 469, 25 (1999)

25. H-Ch. Schröder et al., Eur. Phys. J. C 21, 473 (2001)

26. PSI proposals R-98.01 and R-06.03, www2.fz-juelich.de/ikp/ exotic-atoms

27. M. Hennebach et al., Eur. Phys. J. A 50, 190 (2014)

28. M. Hennebach et al., Eur. Phys. J. A 55, 24(E) (2019)

29. Th Strauch et al., Eur. Phys. J. A 47, 88 (2011)

30. U.C. Bergmann, K. Riisager, Nucl. Instrum. Methods A 489, 444 (2002)

31. R.D. Cousins, Am. J. Phys. 63, 398 (1995)

32. D.S. Covita et al., Phys. Rev. Lett. 102, 023401 (2009)

33. D.S. Covita et al., Eur. Phys. J. D 72, 72 (2018)

34. M. Vardanyan, R. Trotta, J. Silk, Mon. Not. R. Astron. Soc. 413, L91 (2011)

35. U.-G. Meißner, U. Raha, A. Rusetsky, Eur. Phys. J. C 35, 349 (2004)

36. P.Zemp, Pionic Hydrogen in QCD $+Q E D$ : Decay width at NNLO, $\mathrm{PhD}$ thesis, University of Bern (2004)

37. J. Spuller et al., Phys. Lett. 67B, 479 (1977)

38. M. Hoferichter, B. Kubis, U.-G. Meißner, Phys. Lett. B 678, 65 (2009)

39. D. Eiras, J. Soto, Phys. Lett. B 491, 101 (2000)

40. S. Deser, L. Goldberger, K. Baumann, W. Thirring, Phys. Rev. 96, 774 (1954)

41. V.E. Lyubovitzkij, A. Rusetski, Phys. Lett. B 494, 9 (2000)

42. J. Gasser et al., Eur. Phys. J. C 26, 13 (2002)

43. V. Bernard, N. Kaiser, U.-G. Meißner, Phys. Lett. B 309, 421 (1993)

44. V. Bernard, N. Kaiser, U.-G. Meißner, Nucl. Phys. A 615, 483 (1997)

45. M. Mojzis, Eur. Phys. J. C 2, 181 (1998)

46. D. Sigg et al., Nucl. Phys. A 609, 310 (1996)

47. T.E.O. Ericson, B. Loiseau, S. Wycech, Phys. Lett. B 594, 76 (2004)

48. E. Matsinos, G. Rasche, J. Mod. Phys. 3, 1369 (2012)

49. E. Matsinos, G. Rasche, Int. J. Mod. Phys. A 28, 1350039 (2013)

50. M. Hoferichter, B. Kubis, U.-G. Meißner, Nucl. Phys. A 883, 18 (2010)

51. V. Baru et al., Nucl. Phys. A 872, 69 (2011)

52. U.-G. Meißner, U. Raha, A. Rusetsky, Eur. Phys. J. C 41, 213 (2005)

53. V. Baru et al., Phys. Lett. B 589, 118 (2004)

54. U.-G. Meißner, U. Raha, A. Rusetsky, Phys. Lett. B 639, 478 (2006)

55. V. Lensky et al., Phys. Lett. B 648, 46 (2007)

56. V. Baru et al., Phys. Lett. B 659, 184 (2008)

57. V. Baru et al., Phys. Lett. B 694, 473 (2011)

58. V. Baru et al., Eur. Phys. J. A 48, 69 (2012)

59. A.H. Rosenfeld, Phys. Rev. 96, 139 (1954)

60. V. Lensky et al., Eur. Phys. J. A 27, 37 (2006)

61. Th Strauch et al., Phys. Rev. Lett. 104, 142503 (2010)
62. M.L. Goldberger, H. Miyazawa, R. Oehme, Phys. Rev. 99, 986 (1955)

63. T.E.O. Ericson, B. Loiseau, A.W. Thomas, Phys. Rev. C 66 , 014005 (2002)

64. V.V. Abaev, P. Metsä, M.E. Sainio, Eur. Phys. J. A 32, 321 (2007)

65. M.L. Goldberger, S.B. Treiman, Phys. Rev. 110, 1178 (1958)

66. H. Pagels, Phys. Rev. 179, 1337 (1969)

67. J.L. Goity, Phys. Lett. B 454, 115 (1999)

68. V. Bernard, L. Elouadrhiri, U.-G. Meißner, J. Phys. G 28, R1 (2002)

69. M. Hoferichter et al., Eur. Phys. J. A 52, 331 (2016)

70. N.M. Kroll, M.A. Ruderman, Phys. Rev. 93, 233 (1954)

71. V. Bernard, N. Kaiser, Ulf.-G. Meißner, Phys. Lett. B 383, 116 (1996)

72. V. Bernard, N. Kaiser, J. Gasser, U.-G. Meißner, Phys. Lett. B 268, 291 (1991)

73. V. Bernard, B. Kubis, U.G. Meißner, Eur. Phys. J. A 25, 419 (2005)

74. J. Gasser, H. Leutwyler, M.E. Sainio, Phys. Lett. B 253, 252 (1991)

75. A. Bottino, F. Donato, N. Fornengo, S. Scopel, Astropart. Phys. 13, $215(2000)$

76. A. Bottino, F. Donato, N. Fornengo, S. Scopel, Astropart. Phys. 18, 205 (2002)

77. J.R. Ellis, K.A. Olive, C. Savage, Phys. Rev. D 77, 065026 (2008)

78. V. Cirigliano, R. Kitano, Y. Okada, P. Tuzon, Phys. Rev. D 80, 013002 (2009)

79. A. Crivellin, M. Hoferichter, M. Procura, Phys. Rev. D 89, 093024 (2014)

80. M.E. Sainio, PiN Newslett. 16, 138 (2002)

81. D. Siemens et al., Phys. Lett. B 770, 27 (2017)

82. C. Ditsche, M. Hoferichter, B. Kubis, U.-G. Meißner, JHEP 1206, $043(2012)$

83. M. Hoferichter, J. Ruiz de Elvira, B. Kubis, U.-G. Meißner, Phys. Rev. Lett. 115, 092301 (2015)

84. M. Pavan, R.A. Arndt, I.I. Strakovsky, R.L. Workman, PiN Newslett. 16, 110 (2002)

85. J. Ruiz de Elvira, M. Hoferichter, B. Kubis, U.-G. Meißner, J. Phys. G. 45, 024001 (2018)

86. J. Stahov, H. Clement, G.J. Wagner, Phys. Lett. B 726, 685 (2013)

87. J.M. Alarcón, J. Martin Camalich, J.A. Oller, Phys. Rev. D 85, 051503(R) (2012)

88. S. Dürr et al., BMW Collaboration. Phys. Rev. Lett. 116, 172001 (2016)

89. Y.B. Yang et al., $\chi$ QCD Collaboration. Phys. Rev. D 94, 0540503 (2016)

90. A. Abdel-Rehim et al., ETM Collaboration. Phys. Rev. Lett. 116, 252001 (2016)

91. Sz. Borsanyi, et al., arXiv:hep-lat/2007.03319v1 (2020)

92. M. Hoferichter, J. Ruiz de Elvira, B. Kubis, U.-G. Meißner, Phys. Lett. B 760, 74 (2016)

93. M. Leon, H.A. Bethe, Phys. Rev. 127, 636 (1962)

94. F.J. Hartmann, Proceedings of Physics of Exotic Atoms on Electromagnetic Cascade and Chemistry, Erice, Italy, 1989 (Plenum Press, New York, 1990) p. 23 and p. 127, , references therein

95. G.Ya. Korenman, V.P. Popov, Muon Cat. Fus. 4, 145 (1989)

96. G.Ya. Korenman, V.P. Popov, G.A. Fesenko, Muon Cat. Fus. 7, 179 (1992)

97. J.S. Cohen, Phys. Rev. A 59, 1160 (1999)

98. J.S. Cohen, Rep. Prog. Phys. 67, 1769 (2004)

99. L. Bracchi, G. Fiorentini, Nuovo Cim. A 43, 9 (1978)

100. V.N. Pomerantsev, V.P. Popov, Phys. Rev. A 73, 040501(R) (2006)

101. T.S. Jensen, V.E. Markushin, Lec. Notes Phys. 627, 37 (2003)

102. V.P. Popov, V.N. Pomerantsev, Phys. Rev. A 86, 052520 (2012)

103. E. Borie, M. Leon, Phys. Rev. A 21, 1460 (1980)

104. J.B. Czirr et al., Phys. Rev. 130, 341 (1963)

105. A. Badertscher et al., Europhys. Lett. 54, 313 (2001) 
106. T.S. Jensen, V.P. Popov, V.N. Pomerantsev, arXiv:0712.3010 (2007)

107. A.J. Rusi el Hassani et al., Z. Phys. A 351, 113 (1995)

108. J.F. Crawford et al., Phys. Rev. D 43, 46 (1991)

109. T.S. Jensen, V.E. Markushin, Eur. Phys. J. D 19, 165 (2002)

110. T.S. Jensen, V.E. Markushin, Eur. Phys. J. D 21, 261 (2002)

111. T.S. Jensen, V.E. Markushin, Eur. Phys. J. D 21, 271 (2002)

112. S. Jonsell, J. Wallenius, P. Froelich, Phys. Rev. A 59, 3440 (1999)

113. E. Lindroth, J. Wallenius, S. Jonsell, Phys. Rev. A 68, 032502 (2003)

114. E. Lindroth, J. Wallenius, S. Jonsell, 69, 059903(E) (2004)

115. S. Kilic, J.-P. Karr, L. Hilico, Phys. Rev. A 70, 042506 (2004)

116. V.E. Markushin, Phys. Rev. A 50, 1137 (1994)

117. E.C. Aschenauer et al., Phys. Rev. A 51, 1965 (1995)

118. V.E. Markushin, ThS Jensen, Hyperfine Interactions 138, 71 (2001)

119. V.P. Popov, V.N. Pomerantsev, Hyperfine Int. 209, 75 (2012)

120. L.M. Simons, Physica Scripta T22, 90 (1988)

121. L.M. Simons, Hyperfine Interactions 81, 253 (1993)

122. H.H. Johann, Z. Phys. 69, 185 (1931)

123. D.E. Gotta, L.M. Simons, Spectrochim. Acta B 120, 9 (2016)

124. S. Schlesser et al., Phys. Rev. C 84, 015211 (2011)

125. J.C. Brice, J. Mat. Sci. 15, 161 (1980)

126. G. Basile et al., Phys. Rev. Lett. 72, 3133 (1994)

127. N. Nelms et al., Nucl. Instr. Meth. A 484, 419 (2002)

128. P. Indelicato et al., Rev. Scient. Instr. 77, 043107 (2006)

129. Bragg crystals were manufactured in collaboration with Carl Zeiss AG, D-73447 Oberkochen, Germany

130. J. Eggs, K. Ulmer, Z. angew. Phys., 20. Band, Heft 2, 118 (1965)

131. S. Biri, L. Simons, D. Hitz, Rev. Sci. Instrum. 71, 1116 (2000)

132. D.F. Anagnostopoulos et al., Nucl. Instr. Meth. A 545, 217 (2005)

133. P. Indelicato, Phys. Rev. Lett. 77, 3323 (1996)

134. G. Douysset, H. Khodja, A. Girard, J.P. Briand, Phys. Rev. E 61, $3015(2000)$

135. A.G. Drentje, A. Girard, D. Hitz, G. Melin, Rev. Sci. Instrum. 71, $623(2000)$
136. P. Amaro et al., Phys. Rev. Lett. 109, 043005 (2012)

137. A. Hirtl, Determination of the Strong Interaction Ground State Width in Pionic Hydrogen, $\mathrm{PhD}$ thesis, TU Wien (2008)

138. D.S. Covita, High-precision spectroscopy of the $3 p-1$ s transition in muonic hydrogen, $\mathrm{PhD}$ thesis, University of Coimbra (2008), http://hdl.handle.net/10316/7521

139. M. Sanchez del Rio, R.J. Dejus, Proc. SPIE Int. Soc. Opt. Eng. 3448, 246 (1998)

140. M. Sanchez del Rio, R.J. Dejus, Proc. SPIE Int. Soc. Opt. Eng. 5536, 171 (2004) http://www.esrf.eu/computing/scientific/xop2. 1

141. D.S. Covita et al., Rev. Sci. Instr. 79, 033102 (2008)

142. F. James, M. Roos, Comput. Phys. Commun. 10, 343 (1975)

143. S. Baker, R.D. Cousins, Nucl. Instr. Meth. 221, 437 (1984)

144. T. Hauschild, M. Jentschel, Nucl. Instr. Meth. A 457, 384 (2001)

145. D. Gotta et al., Lect. Notes Phys. 745, 165 (2008)

146. R. Trotta, Contemporary Physics 49(2), 71 (2008)

147. M. Hoferichter, private communication

148. D.V. Bugg, Eur. Phys. J. C 33, 505 (2004)

149. R.A. Arndt et al., Phys. Rev. C 74, 045205 (2006)

150. J.J. deSwart, M.C.M. Rentmeester, R.G.E. Timmermans, PiN Newsl. 13, 96 (1997)

151. N.F. Nasrallah, Phys. Rev. D 62, 036006 (2002)

152. R. Navarro Pérez, J.E. Amaro, E. Ruiz Arriola, Phys. Rev. C 95, 064001 (2017)

153. P. Reinert, H. Krebs, E. Epelbaum, arXiv:nucl-th/2006.15360v1 (2020)

154. M.A. Kovash et al., PiN Newsl. 12, 51 (1997)

155. O. Hanstein, D. Drechsel, L. Tiator, Nucl. Phys. A 632, 561 (1998)

156. B. Märkisch et al., Phys. Rev. Lett. 122, 242501 (2019)

157. R. Koch, E. Pietarinen, Nucl. Phys. A 336, 331 (1980)

158. G. Höhler, Pion-Nukleon-Streuung: Methoden und Ergebnisse (Landolt-Börnstein vol 9b2), ed. H. Schopper (Berlin: Springer 1983) 